\title{
Analyse des représentations sociales des enseignants.es du « produire autrement » vis-à-vis des directives ministérielles
}

Nina Asloum et Laurent Bedoussac

\section{(2) OpenEdition Journals}

Édition électronique

URL : http://journals.openedition.org/ere/5788

DOI : 10.4000/ere.5788

ISSN : 2561-2271

Éditeur

Centr'ERE

\section{Référence électronique}

Nina Asloum et Laurent Bedoussac, « Analyse des représentations sociales des enseignants.es du " produire autrement » vis-à-vis des directives ministérielles », Éducation relative à l'environnement [En ligne], Volume 15 - 2 | 2020, mis en ligne le 15 novembre 2020, consulté le 22 février 2021. URL : http:// journals.openedition.org/ere/5788; DOI : https://doi.org/10.4000/ere.5788 


\title{
Analyse des représentations sociales des enseignants.es $\mathrm{du}$ " produire autrement » vis-à-vis des directives ministérielles
}

\author{
Nina Asloum et Laurent Bedoussac
}

1 L'enseignement agricole, dont les établissements couvrent l'ensemble du territoire national, est le deuxième système éducatif public en France avec plus de 200000 élèves, apprentis et étudiants répartis dans 807 établissements, 136 centres de formation d'apprentis et 19 écoles d'enseignement supérieur agricole (Ministère de l'agriculture et de l'alimentation, 2019a). Fortement ancré dans le monde professionnel, l'enseignement agricole prépare les élèves aux métiers de la nature et du vivant tout en assurant la formation des agriculteurs et des acteurs du monde rural. Il est bien souvent considéré comme un lieu d'innovation pédagogique du fait de son histoire et de sa réactivité face aux demandes sociétales émergentes (Boulet, 2003).

2 L'agriculture intensive, prônée dès les années 50, est aujourd'hui fortement décriée notamment en raison de ses impacts sur l'environnement. Ainsi, dans ses travaux préparatoires de la Loi d'avenir pour l'agriculture, l'alimentation et la forêt (Loi $n^{\circ}$ 2014-1170), le Ministère de l'Agriculture et de l'Alimentation a opéré un changement qui vise à réviser en profondeur les modèles de développement en agriculture vers la transition agroécologique. Pour l'enseignement agricole cela s'est traduit par l'inscription dans l'article premier du code rural et de la pêche maritime du 13 octobre 2014, d'un projet stratégique national qui l'engage dans une voie de réforme de son système de formation devant prendre en compte les transitions à la fois écologiques, pédagogiques et éducatives; ainsi sont apparues les expressions "produire autrement » et « enseigner à produire autrement ».

3 Face à ces nouvelles prescriptions, les enseignants.es manifestent des difficultés tout d'abord, dans la définition des contours de l'agroécologie et du " produire autrement ", et ensuite dans la transposition didactique des savoirs et des pratiques sociales de 
référence (Martinand, 1989). Ces difficultés sont d'autant plus exacerbées que ces savoirs sont pluriels (académiques, professionnels, sociaux, etc.) et le plus souvent en construction et incertains comparativement à ceux que les enseignants.es ont pour habitude de mobiliser. Ces difficultés sont également liées au positionnement des enseignants.es face à deux disciplines que sont l'agronomie et l'écologie, souvent considérées comme étant à la base de l'agroécologie (Stassart et coll., 2012).

De nombreux travaux concernant l'éducation au développement durable (EDD) ont mis en évidence le manque de formation des enseignants.es (dont Girault et coll., 2007 ; Lange, 2011). En particulier, Bouillier-Oudot et Asloum (2014) ont relevé la nécessité d'un « temps didactique » pour permettre de traduire ces nouveaux concepts en objets d'enseignement compréhensibles et transférables. Ainsi, à l'heure où le plan « enseigner à produire autrement » est entré dans la sphère éducative, l'École Nationale Supérieure de Formation de l'Enseignement Agricole (ENSFEA), en tant qu'organisme de formation professionnelle initiale et continue des enseignants.es, a proposé et mis en œuvre dès 2014 une démarche pédagogique pluridisciplinaire (conférences, ateliers, débats et visites d'exploitations agricoles) pour accompagner les enseignants.es lauréats.es des concours externes et internes tout au long de leur année de formation.

Legardez (2004) rappelle que les représentations sociales des enseignants.es influencent leurs pratiques éducatives et peuvent affecter leurs postures citoyennes. Selon Garnier et Sauvé (1999), partir de l'analyse des représentations sociales peut donc s'avérer une stratégie prometteuse, d'autant plus qu'elle peut s'inscrire dans une perspective réflexive en vue de contribuer à la transformation des pratiques sociales. En nous appuyant sur l'hypothèse que les représentations sociales individuelles des enseignants.es du "produire autrement " sont a priori étroites, nous avons procédé à une analyse de celles-ci dans le but de les faire évoluer et de permettre aux enseignants.es de construire une vision commune et partagée. Les résultats de cette étude exploratoire visent à éclairer certaines difficultés rencontrées par les enseignants.es et à identifier des pistes pour leur formation.

Dans cet article, nous présenterons dans un premier temps le contexte et les enjeux institutionnels auxquels l'enseignement agricole est confronté aujourd'hui. Puis, après une analyse synthétique des contours scientifiques couverts par l'agroécologie, nous présenterons le cadre théorique et méthodologique mobilisé. Pour finir, nous nous attacherons à comprendre, par la méthode de l'approche structurale des représentations sociales selon la théorie du noyau central (Abric, 1994), comment les spécificités disciplinaires interagissent dans la mise en œuvre de cette nouvelle injonction.

\section{Le contexte institutionnel : du global au local}

7 La première conférence des Nations unies sur l'environnement humain à Stockholm en 1972, a marqué une évolution décisive dans le rapport qu'entretient la société avec son environnement, ce qui s'est traduit depuis par l'intégration progressive du développement durable dans les politiques publiques. A partir des années 80 , le modèle productiviste devient la cible de nombreuses critiques notamment dans la publication du rapport Poly (1978) dont les conclusions mettent en exergue la nécessité de réorienter l'agriculture vers un développement durable en opposition à l'intensification et la spécialisation de la production agricole qui occasionnent des 
dommages environnementaux et sociaux et un accroissement des dépenses publiques (Allaire et Boyer, 1995). Ces critiques ont contraint la politique agricole commune européenne à engager progressivement une série de réformes pour redéfinir l'activité des agriculteurs avec notamment l'instauration des quotas laitiers en 1984 et des mesures agri-environnementales en 1985 ouvrant la possibilité d'accorder des primes aux agriculteurs mettant en œuvre des pratiques respectueuses de l'environnement (Muller, 2000). Cette période témoigne d'un tournant de la politique agricole commune en ce qui concerne les relations entre agriculture et environnement, avec une convergence des impératifs écologiques et économiques.

8 La volonté de reconnaître l'éducation dans l'enseignement agricole français, comme un moyen important pour la préservation de l'environnement à long terme, remonte aux années 1970 avec la création en 1971 de la première et unique formation de technicien supérieur "protection de la nature " (Mabit, 1991). Le fait nouveau pour ce diplôme réside dans l'introduction de l'écologie en tant que discipline scientifique et sa mobilisation dans la dimension technique des apprentissages. En pénétrant le champ de l'aménagement des espaces, l'écologie redessine le contour des pratiques professionnelles et la façon de penser les relations entre société et nature. Le basculement du développement durable de sa sphère onusienne d'origine vers une autre sphère, celle de l'école, "montre de nouvelles missions" assignées à cette dernière au sens de Martinand (2003). En 1985, la Direction Générale de l'Enseignement et de la Recherche (DGER) entreprend une rénovation complète de l'ensemble de ses formations pour prendre en compte les préoccupations sociales en matière d'environnement. L'enseignement agricole est concerné à double titre, en tant que dispositif de formation professionnelle initiale et continue des acteurs de la production agroalimentaire et de la gestion de l'espace rural et au travers de sa mission éducative. La DGER précise d'ailleurs aux enseignants.es que "l'ambition de l'enseignement agricole est de former un professionnel compétent et un futur citoyen en lui donnant les moyens de comprendre le monde qui l'entoure et d'agir, de communiquer selon les valeurs démocratiques de notre société » (Ministère de l'Agriculture et de l'Alimentation, 2020).

9 Cependant, il faudra attendre quatorze années pour qu'un premier cadrage réglementaire généralise l'EDD dans le système éducatif français par la circulaire $\mathrm{n}^{\circ}$ 2004-110. En 2007, la seconde phase de généralisation de l'EDD est engagée via la circulaire n 2007-077 - reprise la même année par la DGER sous la forme d'une circulaire propre à l'enseignement agricole - et la troisième phase voit le jour en 2011 avec la circulaire $n^{\circ}$ 2011-186 prolongée jusqu'en 2030 par la circulaire $n^{\circ} 2019-12$ de 2019. En 2014, le projet agro-écologique pour la France est initié, trouvant ses fondements auprès d'auteurs tels que Altieri et Gliessman qui, dès les années 80, considèrent l'agroécologie comme un ensemble de méthodes et de pratiques permettant de réviser les liens entre agriculture et écosystèmes dans le but de garantir la préservation des ressources naturelles (Altieri, 1987; Gliessman, 1990). De nouvelles directives, émanant du Ministère de l'Agriculture et de l'Alimentation, accompagnent ce projet avec le lancement en 2014 du plan d'action «enseigner à produire autrement» pour l'enseignement agricole, renforcé en 2019 par de nouvelles instructions dans le cadre du plan EPA2 «enseigner à produire autrement pour les transitions et l'agroécologie ». 
Ces différents textes de cadrage ont largement défini les orientations politiques et les instructions pour l'éducation relative à l'environnement (ERE) puis pour l'EDD selon un triple changement sur les plans éthique, scientifique et politique (Asloum et Kalali, 2013) comme en témoigne par exemple le référentiel de diplôme du baccalauréat professionnel « conduite et gestion de l'exploitation agricole » préparant aux métiers d'agriculteur et de conseiller en agriculture (Ministère de l'Agriculture et de l'Alimentation, 2019b) qui précise :

De l'agroécosystème au système alimentaire mondial, la «triple performance » économique, sociale et environnementale et la transition agro-écologique constituent le nouveau modus operandi de la conduite et de l'accompagnement du changement dans les exploitations agricoles.

11 L'enseignement doit donc permettre de faire changer les pratiques agricoles car, comme le souligne ce même document prescriptif, le bilan reste mitigé montrant que l'ambition affichée ne va pas de soi :

Si des changements de pratiques importants ont déjà été réalisés ces dernières années, beaucoup reste à faire compte tenu de la montée des attentes des consommateurs et des citoyens en matière d'éthique, de développement durable et de bien-être animal.

12 Au-delà des missions de transmission de connaissances disciplinaires et de compétences transversales, l'enseignement agricole est sollicité par une action éducative plus large : former de futurs citoyens responsables. Il s'agit de développer une pensée critique chez les élèves afin qu'ils leur permettent de participer et d'agir dans le monde actuel et incertain à venir (Lange, 2015). Notre recherche s'inscrit donc dans la mouvance de la théorie critique de l'ERE et de l'EDD où de nombreux chercheurs conviennent de l'importance d'une éducation émancipatrice, engageant les élèves dans un positionnement réflexif et critique vis à vis des idéologies et des normes sociales (Sauvé, 1999 ; Girault et coll., 2007 ; Lange, 2015 ; Barthes, 2017).

\section{L'agroécologie : un concept polysémique}

13 Selon Gliessman (1990), l'agroécologie consiste à proposer des modes de production reposant sur l'utilisation des principes et des concepts issus de l'écologie, reconnaissant ainsi l'importance de la biodiversité et des processus naturels associés (cycle des éléments et équilibres biologiques entre organismes). Dès lors, elle apparaît comme une alternative à l'agriculture dite conventionnelle fondée sur l'artificialisation des cultures par l'usage d'intrants de synthèse (engrais, pesticides) et d'énergies fossiles (Hazard et coll., 2016). D'après Tomich et coll. (2011), l'agroécologie peut donc être définie comme « un ensemble disciplinaire alimenté par l'hybridation des sciences agronomiques (agronomie, zootechnie), de l'écologie appliquée aux agroécosystèmes et des sciences humaines et sociales (sociologie, économie, géographie)». Toutefois, elle peut être considérée dans une acception plus large intégrant l'ensemble du système alimentaire, pour permettre la transition vers des systèmes viables et respectueux des humains et de leur environnement. Ainsi, Francis et coll. (2003) définissent l'agroécologie comme "l'étude intégrative de l'écologie de l'ensemble du système alimentaire, intégrant les dimensions écologiques, économiques et sociales ».

14 Cet élargissement du concept d'agroécologie nécessite de considérer les dynamiques des systèmes territoriaux et les acteurs (Wezel et coll., 2009), permettant l'émergence 
de modes de production et de filières qui valorisent les potentialités des territoires selon les trois piliers de la durabilité (écologique, économique et social). Le développement de tels systèmes alimentaires nécessite donc de s'appuyer sur des approches transdisciplinaires et multi-acteurs (professionnels du secteur agricole, scientifiques, politiques publiques et acteurs des mouvements sociaux). Dès lors, le positionnement scientifique de l'agroécologie se réfère autant à des pratiques agricoles qu'à un mouvement social selon Wezel et coll. (2009). D'ailleurs, dans la sphère publique, l'agroécologie est défendue par des acteurs prônant la souveraineté alimentaire et l'agriculture paysanne, et interrogeant dès lors notre manière de consommer, notamment par le développement de circuits courts recréant des liens entre production et consommation. Ces différents éléments questionnent donc l'organisation actuelle des systèmes agroalimentaires au regard de leur durabilité et appellent à reconsidérer les liens entre agriculture, environnement, science, société et politique.

De par sa nature polysémique et ses contours flous, «il n'existe pas une seule manière de définir et de travailler sur l'agroécologie » comme le soulignent Stassart et coll. (2012). Nous avons donc souhaité analyser les représentations sociales des enseignants.es et questionner l'impact de leur identité disciplinaire au regard de la diversité de ces acceptions.

\section{Cadre théorique des représentations sociales}

Les représentations sociales peuvent être appréhendées et vues comme " un ensemble d'informations, de croyances, d'opinions et d'attitudes à propos d'un objet donné " (Abric, 2005). De même, pour Jodelet (2003), la représentation sociale est « une forme de connaissance, socialement élaborée et partagée, ayant une visée pratique et concourant à la constitution d'une réalité commune à un ensemble social» qui se construit invariablement au sein d'un groupe et sur un objet donné car «il n'y a pas de représentation sans objet ». L'émergence d'une représentation sociale doit remplir trois conditions :

- La première condition concerne la dispersion de l'information au sujet de l'objet.

Les informations sur l'objet social peuvent être insuffisantes ou surabondantes, souvent en raison de la nature de l'objet, de sa complexité, de la difficulté d'accès à l'information et de l'insuffisance des connaissances nécessaires pour l'appréhender. On parle alors de " dispersion de l'information » pouvant entraîner un décalage et de nombreuses distorsions par rapport aux informations dont on dispose réellement (Moliner, 1993).

- La seconde condition porte sur le principe de focalisation.

Selon Moscovisi (1961), le « principe de focalisation » est relatif à un groupe ou à un individu, chacun pouvant choisir de s'attacher seulement à certains aspects de l'objet sans pour autant l'appréhender dans sa globalité selon leur intérêt ou de leur implication.

- La troisième condition repose sur le principe de pression à l'inférence. 
Du fait des rapports sociaux du groupe (la position, la reconnaissance de l'individu dans le groupe ou la pression exercée par le groupe), la représentation sociale est à la fois la reproduction du sens commun et la production d'un sens nouveau qui peut être partagé dans le groupe (Abric, 2005), ce que Moscovisi (1961) qualifie de « pression à l'inférence ». Ainsi, les représentations sociales contribuent à la construction d'une réalité commune qui s'élabore dans un contexte social dans lequel l'individu est amené à adhérer au groupe pour s'approprier l'objet de la représentation dans un rapport de symbolisation et d'interprétation (Jodelet, 1989).

Cherchant à comprendre les représentations sociales des enseignants.es sur le «produire autrement» - pris comme un objet social polymorphe -, nous avons mobilisé la théorie du noyau central et des éléments périphériques (Abric, 1994 ; Flament, 1994a, 1994b ; Vergès, 1994). Abric (1994) - prenant en partie appui sur le processus d'objectivation - insiste sur l'importance de la structuration des représentations sociales, considérant que «l'analyse d'une représentation, la compréhension de son fonctionnement nécessite obligatoirement un double repérage : celui de son contenu et celui de sa structure "; il fait ici l'hypothèse que "non seulement les éléments de la représentation sociale sont hiérarchisés, mais [que] par ailleurs toute représentation est organisée autour d'un noyau central constitué d'un ou de quelques éléments qui donnent à la représentation sa signification ».

Selon Abric (2005), l'étude de l'organisation interne des représentations sociales à partir de l'approche structurale permet de prendre en compte un ensemble d'éléments hiérarchisés en distinguant deux zones : (1) le noyau central - élément structurant et stable - qui détermine la significativité et la permanence de la représentation et qui est porteur de consensus et autour duquel s'organisent (2) les éléments périphériques qui sont hiérarchisés et permettent à la fois le maintien de la représentation et son adaptabilité aux différents contextes. Ainsi, alors que le noyau central résiste aux changements en étant fortement marqué par la mémoire collective du groupe et associé aux valeurs et aux normes sociales, les éléments périphériques ont quant à eux un caractère plus souple car liés aux caractéristiques individuelles et à l'environnement immédiat. Par ailleurs, la position des éléments périphériques, plus ou moins proche du noyau central, joue un rôle important dans la significativité de la représentation. Selon cette théorie, deux représentations seront différentes si et seulement si elles sont organisées autour de deux noyaux distincts. Dès lors, repérer le contenu de la représentation ne suffit pas car il est nécessaire d'appréhender son organisation par l'analyse du noyau central, des éléments périphériques et des relations entre les contenus informationnels. Pour ce faire, nous avons eu recours au logiciel IRaMuTeQ (Interface de R pour les Analyses Multidimensionnelles de Textes et de Questionnaires) en considérant que l'analyse des représentations sociales pourrait permettre d'avancer des hypothèses sur les processus de transformation de celles-ci.

\section{Méthodologie}

19 Notre méthode de recueil des données s'appuie sur une série d'évocations libres et hiérarchisées (Abric, 2005) relatives à l'expression "produire autrement » que nous avons soumis à cinq cohortes d'enseignants.es. Cette activité s'inscrit dans le cadre 
d'une démarche pédagogique pluridisciplinaire mise en œuvre par l'ENSFEA à partir de 2014 pour la formation professionnelle des enseignants.es. Ce recueil des données a été réalisé en trois phases : (1) un recueil individuel des trois mots qui définissent le mieux la notion de "produire autrement ", (2) un travail collectif par groupe de cinq à huit enseignants.es de différentes disciplines devant se mettre d'accord sur six mots clés résumant la notion de "produire autrement " et (3) une phase au cours de laquelle chaque enseignant.e doit répartir cinq votes pour les notions les plus représentatives $\mathrm{du}$ « produire autrement » parmi celles identifiées lors de la deuxième phase.

\section{Population}

Le recueil des données a été réalisé au cours de cinq séances organisées entre le 16/06/2014 et le 17/11/2015 avec pour chacune d'elles entre 53 et 87 enseignants.es, soit une population totale de 340 enseignants.es dont 200 femmes ( $58,8 \%$ des effectifs). Sur cette population, 117 enseignants.es $(34,4 \%)$ sont issus des concours externes de l'enseignement agricole et seront qualifiés par la suite de "novice " alors que les autres enseignants.es sont issus des concours internes et seront regroupés sous le qualificatif «expérimenté ». En terme d'appartenance disciplinaire, 34,0\% des enseignants.es relèvent de disciplines techniques et professionnelles (génie alimentaire, aménagement des espaces naturel, aménagement paysager, agroéquipement, productions animales, animalerie, aquaculture, hippologie, productions horticoles, productions végétales, vigne et vin) et seront regroupés sous le terme « technique ». Les autres enseignants.es, regroupés.es sous la dénomination "non technique", associés pour $44,1 \%$ à des disciplines de sciences humaines et sociales (éducation socio-culturelle, histoiregéographie, lettres-histoire, lettres modernes, langues vivantes, documentation) dont $16,5 \%$ aux sciences économiques et sociales et gestion (gestion de l'entreprise, économie sociale et familiale, gestion commerciale, gestion de l'exploitation agricole), pour 15,6\% à des disciplines scientifiques (biologie-écologie, biochimie microbiologie et biotechnologie, mathématiques, physique-chimie) et pour 5,9\% à d'autres disciplines (éducation physique et sportive ; technologie de l'informatique et du multimédia).

\section{Procédure de recueil des données}

21 Notre méthode s'appuie sur une série d'évocations sur le " produire autrement » à la fois : (1) évocations libres, ce qui revient à indiquer de manière spontanée tous les mots ou expressions qui viennent à l'esprit pour accéder rapidement et simplement aux éléments constitutifs de l'univers sémantique de l'objet étudié et (2) évocations hiérarchisées, ce qui consiste à classer les réponses en fonction de l'importance accordée à chaque terme pour définir l'objet en question. Cette stratégie permet d'accéder au discours spontané sur l'objet de la représentation et de déterminer l'univers sémantique d'une population donnée. Il s'agit en particulier de repérer le vocabulaire associé à la notion étudiée, ici " produire autrement » et de saisir les sens que les individus accordent à cette notion. Cette méthodologie permet in fine de donner une première information sur la structure de la représentation et son organisation. Dans le cas présent, le recueil des données s'est déroulé en trois temps correspondant à trois activités distinctes. 


\section{Activité 1 : représentation individuelle de " produire autrement "} pour garantir une composition pluridisciplinaire. Chaque groupe doit se mettre d'accord sur six mots clés qui, par ordre d'importance, résument la notion de "produire autrement ». Le corpus constitué par cette activité sur l'ensemble des cinq séances comporte 300 mots ou expressions ( 50 groupes sur l'ensemble des cinq sessions et six mots par groupe).

\section{Activité 3 : représentation collective de " produire autrement "}

Après avoir supprimé les doublons au sein des mots obtenus lors de l'activité 2 , une liste triée par ordre alphabétique est présentée aux enseignants.es, à qui nous demandons de répartir individuellement cinq votes pour les notions les plus représentatives $\mathrm{du}$ " produire autrement ». Les enseignants.es sont appelés à ne pas de communiquer entre eux et le corpus constitué par cette activité sur l'ensemble des cinq séances comporte 1700 mots ou expressions (340 enseignants.es et cinq mots par enseignant.e).

\section{Catégorisation des réponses}

A l'issu des cinq séances, les corpus ont été harmonisés (adjectifs mis au masculin singulier, verbes mis à l'infinitif, suppression des articles, suppression des accents, suppression des majuscules, suppression des abréviations, etc.). In fine, chaque activité a donné lieu à un corpus contenant 452, 172 et 165 termes différents respectivement pour les activités 1,2 et 3 . A noter que seuls les corpus des activités 1 et 3 seront analysés. En effet, l'activité 2 constitue une étape intermédiaire dont la fonction principale était d'élargir la représentation des enseignants.es $\mathrm{du}$ "produire autrement » par un dialogue entre pairs.

En raison du nombre élevé de termes générés lors des activités 1 et 3 nous avons procédé à un regroupement en 21 catégories (Figures $1 \mathrm{abc}$ ). Par exemple la catégorie changement, représentant $10,8 \%$ des occurrences sur les activités 1 et 3 , se réfère par ordre décroissant d'importance à: innovation, innover, changement, changement [de] mentalité, progrès, etc.). À noter que cette catégorisation a été réalisée d'abord indépendamment par chacun des deux chercheurs (auteurs de cet article) avant une phase de concertation visant à harmoniser leurs points de vue. 
Figure 1a

\begin{tabular}{|c|c|c|c|c|c|c|c|}
\hline \multirow[t]{2}{*}{$\begin{array}{l}\text { CATEGORIE } \\
\text { TERME }\end{array}$} & \multicolumn{3}{|c|}{$\begin{array}{c}\text { Pourcentage d'occurrence } \\
\text { par activité }\end{array}$} & \multirow[t]{2}{*}{$\begin{array}{l}\text { CATEGORIE } \\
\text { TERME }\end{array}$} & \multicolumn{3}{|c|}{$\begin{array}{c}\text { Pourcentage d'occurrence } \\
\text { par activité }\end{array}$} \\
\hline & 1 & 3 & 1 et 3 & & 1 & 3 & 1 et 3 \\
\hline changement & $10.49 \%$ & $10.94 \%$ & $10.77 \%$ & durable & $9.31 \%$ & $9.82 \%$ & $9.63 \%$ \\
\hline innovation & $1.37 \%$ & $4.24 \%$ & $3.16 \%$ & duratilite & $2.55 \%$ & $5.29 \%$ & $4.26 \%$ \\
\hline innover & $1.37 \%$ & $0.94 \%$ & $1.10 \%$ & developpement_durable & $4.22 \%$ & $2.59 \%$ & $3.20 \%$ \\
\hline changement & $0.98 \%$ & $0.82 \%$ & $0.88 \%$ & durable & $2.16 \%$ & $1.94 \%$ & $2.02 \%$ \\
\hline changement_mentalite & $0.00 \%$ & $1.35 \%$ & $0.85 \%$ & education & $4.31 \%$ & $12.29 \%$ & $9.30 \%$ \\
\hline progges & $0.29 \%$ & $0.41 \%$ & $0.37 \%$ & respensabilite & $0.29 \%$ & $1.47 \%$ & $1.03 \%$ \\
\hline changement_mode_consommination & $0.00 \%$ & $0.59 \%$ & $0.37 \%$ & citoyennete & $0.10 \%$ & $1.29 \%$ & $0.85 \%$ \\
\hline produire_moins_produire_mieux & $0.00 \%$ & $0.53 \%$ & $0.33 \%$ & transmission & $0.00 \%$ & $1.12 \%$ & $0.70 \%$ \\
\hline urgence & $0.00 \%$ & $0.53 \%$ & $0.33 \%$ & education & $0.10 \%$ & $1.06 \%$ & $0.70 \%$ \\
\hline experimentation & $0.29 \%$ & $0.35 \%$ & $0.33 \%$ & eduquer ehangement & $0.00 \%$ & $1.06 \%$ & $0.66 \%$ \\
\hline changer & $0.00 \%$ & $0.47 \%$ & $0.29 \%$ & responsable & $0.39 \%$ & $0.53 \%$ & $0.48 \%$ \\
\hline changement_pratique & $0.29 \%$ & $0.24 \%$ & $0.26 \%$ & ecocitoyennete & $0.10 \%$ & $0.59 \%$ & $0.40 \%$ \\
\hline environnement & $12.25 \%$ & $9.12 \%$ & $10.29 \%$ & reflexion & $0.59 \%$ & $0.29 \%$ & $0.40 \%$ \\
\hline biodiversitc & $2.06 \%$ & $3.24 \%$ & $2.79 \%$ & respensabiliser & $0.10 \%$ & $0.53 \%$ & $0.37 \%$ \\
\hline respect_enviromnement & $1.08 \%$ & $1.29 \%$ & $1.21 \%$ & communication & $0.00 \%$ & $0.47 \%$ & $0.29 \%$ \\
\hline environnement & $1.57 \%$ & $0.65 \%$ & $0.99 \%$ & informer & $0.10 \%$ & $0.41 \%$ & $0.29 \%$ \\
\hline ressource & $0.29 \%$ & $0.53 \%$ & $0.44 \%$ & promouvoir_sensibiliser & $0.00 \%$ & $0.41 \%$ & $0.26 \%$ \\
\hline preservation_ressource & $0.20 \%$ & $0.47 \%$ & $0.37 \%$ & & & & \\
\hline Irespecl_ccosystcmc_vivant & $0.00 \%$ & $0.53 \%$ & $0.33 \%$ & & & & \\
\hline naturel & $0.39 \%$ & $0.18 \%$ & $0.26 \%$ & & & & \\
\hline preservation_ressource_naturel & $0.00 \%$ & $0.41 \%$ & $0.26 \%$ & & & & \\
\hline
\end{tabular}

Figure 1b

\begin{tabular}{|c|c|c|c|c|c|c|c|}
\hline \multirow[t]{2}{*}{$\begin{array}{l}\text { CATEGORIE } \\
\text { TERME }\end{array}$} & \multicolumn{3}{|c|}{$\begin{array}{c}\text { Pourcentage d'occurrence } \\
\text { par activité }\end{array}$} & \multirow[t]{2}{*}{$\begin{array}{l}\text { CATEGORIE } \\
\text { TERME }\end{array}$} & \multicolumn{3}{|c|}{$\begin{array}{c}\text { Pourcentage d'occurrence } \\
\text { par activité }\end{array}$} \\
\hline & 1 & 3 & 1 et 3 & & 1 & 3 & 1 et 3 \\
\hline technique & $12.65 \%$ & $5.18 \%$ & $7.98 \%$ & valeur & $4.12 \%$ & $7.06 \%$ & $5.96 \%$ \\
\hline raisonner & $1.37 \%$ & $0.71 \%$ & $0.96 \%$ & respect & $1.27 \%$ & $3.65 \%$ & $2.76 \%$ \\
\hline gerer_ressource & $0.10 \%$ & $0.71 \%$ & $0.48 \%$ & mentalite & $0.29 \%$ & $0.82 \%$ & $0.63 \%$ \\
\hline production_raisonner & $0.29 \%$ & $0.47 \%$ & $0.40 \%$ & solidaritc & $0.10 \%$ & $0.59 \%$ & $0.40 \%$ \\
\hline efficacitc & $0.69 \%$ & $0.24 \%$ & $0.40 \%$ & partager & $0.39 \%$ & $0.35 \%$ & $0.37^{t}$ \\
\hline solution_altematif_innovation & $0.00 \%$ & $0.65 \%$ & $0.40 \%$ & valoriser & $0.00 \%$ & $0.53 \%$ & $0.33 \%$ \\
\hline efficient & $0.20 \%$ & $0.41 \%$ & $0.33 \%$ & valorisation & $0.10 \%$ & $0.41 \%$ & $0.29 \%$ \\
\hline technique_alternatif & $0.20 \%$ & $0.29 \%$ & $0.26 \%$ & produire_mieux_pour_monde & $0.10 \%$ & $0.35 \%$ & $0.26 \%$ \\
\hline territoire & $5.69 \%$ & $7.94 \%$ & $7.10 \%$ & alimentation & $5.39 \%$ & $4.29 \%$ & $4.71 \%$ \\
\hline territoire & $0.59 \%$ & $1.65 \%$ & $1.25 \%$ & qualite & $2.16 \%$ & $1.82 \%$ & $1.95 \%$ \\
\hline local & $1.76 \%$ & $0.76 \%$ & $1.14 \%$ & consommer_autrement & $0.29 \%$ & $0.71 \%$ & $0.55 \%$ \\
\hline penser_globalemenent_agir_localement & $0.00 \%$ & $1.00 \%$ & $0.63 \%$ & enjeu_securite_alimentaire & $0.00 \%$ & $0.65 \%$ & $0.40 \%$ \\
\hline circuit local & $0.00 \%$ & $0.82 \%$ & $0.51 \%$ & alimentation & $0.29 \%$ & $0.35 \%$ & $0.33 \%$ \\
\hline territoire_pattrimoine & $0.00 \%$ & $0.65 \%$ & $0.40 \%$ & securite_alimentaire & $0.10 \%$ & $0.47 \%$ & $0.33 \%$ \\
\hline circuit_court & $0.39 \%$ & $0.35 \%$ & $0.37 \%$ & savoir & $5.69 \%$ & $3.88 \%$ & $4.56 \%$ \\
\hline produire_localement & $0.00 \%$ & $0.47 \%$ & $0.29 \%$ & ecologie & $3.63 \%$ & $1.41 \%$ & $2.24 \%$ \\
\hline \multirow[t]{4}{*}{ vision globale raisonner } & $0.10 \%$ & $0.35 \%$ & $0.26 \%$ & ceonomic & $0.78 \%$ & $1.29 \%$ & $1.10 \%$ \\
\hline & & & & recherche_innovation & $0.00 \%$ & $0.65 \%$ & $0.40 \%$ \\
\hline & & & & agroecologie & $4.31 \%$ & $4.41 \%$ & $4.38 \%$ \\
\hline & & & & agroecologie & $4.31 \%$ & $4.41 \%$ & $4.38 \%$ \\
\hline
\end{tabular}




\begin{tabular}{|c|c|c|c|c|c|c|c|}
\hline \multirow[t]{2}{*}{$\begin{array}{l}\text { CATEGORIE } \\
\text { TERME }\end{array}$} & \multicolumn{3}{|c|}{$\begin{array}{c}\text { Pourcentage d'occurrence } \\
\text { par activité }\end{array}$} & \multirow[t]{2}{*}{$\begin{array}{l}\text { CATEGORIE } \\
\text { TERME }\end{array}$} & \multicolumn{3}{|c|}{$\begin{array}{c}\text { Pourcentage d'occurrence } \\
\text { par activité }\end{array}$} \\
\hline & 1 & 3 & 1 et 3 & & 1 & 3 & 1 et 3 \\
\hline bien_ctre & $1.76 \%$ & $5.41 \%$ & $4.04 \%$ & avenir & $2.16 \%$ & $3.59 \%$ & $3.05 \%$ \\
\hline sante & $0.69 \%$ & $3.12 \%$ & $2.21 \%$ & avenir & $0.69 \%$ & $2.06 \%$ & $1.54 \%$ \\
\hline bien_etre & $0.29 \%$ & $1.47 \%$ & $1.03 \%$ & $\operatorname{defi}$ & $0.00 \%$ & $0.88 \%$ & $0.55 \%$ \\
\hline bien_ctre_animal & $0.29 \%$ & $0.53 \%$ & $0.44 \%$ & economie & $3.92 \%$ & $1.65 \%$ & $2.50 \%$ \\
\hline social & $3.63 \%$ & $3.71 \%$ & $3.68 \%$ & viattilite & $0.20 \%$ & $0.82 \%$ & $0.59 \%$ \\
\hline homme & $0.10 \%$ & $0.88 \%$ & $0.59 \%$ & rentabilite & $0.39 \%$ & $0.24 \%$ & $0.29 \%$ \\
\hline equilibre & $0.29 \%$ & $0.53 \%$ & $0.44 \%$ & alternatif & $1.18 \%$ & $3.00 \%$ & $2.32 \%$ \\
\hline engagement & $0.10 \%$ & $0.53 \%$ & $0.37 \%$ & penser_autrement & $0.10 \%$ & $2.35 \%$ & $1.51 \%$ \\
\hline cyuilable & $0.29 \%$ & $0.41 \%$ & $0.37 \%$ & altcmatif & $0.29 \%$ & $0.65 \%$ & $0.51 \%$ \\
\hline equite_social & $0.00 \%$ & $0.47 \%$ & $0.29 \%$ & diversite & $1.86 \%$ & $1.06 \%$ & $1.36 \%$ \\
\hline agriculture & $5.59 \%$ & $1.88 \%$ & $3.27 \%$ & diversite & $0.69 \%$ & $0.71 \%$ & $0.70 \%$ \\
\hline agriculture_hiologique & $3.04 \%$ & $0.94 \%$ & $1.73 \%$ & diversifier & $0.39 \%$ & $0.29 \%$ & $0.33 \%$ \\
\hline agiculture raisumer & $0.69 \%$ & $0.47 \%$ & $0.55 \%$ & adaptation & $1.18 \%$ & $1.06 \%$ & $1.10 \%$ \\
\hline agriculture_durable & $0.29 \%$ & $0.41 \%$ & $0.37 \%$ & adapter & $0.20 \%$ & $0.35 \%$ & $0.29 \%$ \\
\hline biologique & $0.78 \%$ & $0.06 \%$ & $0.33 \%$ & adaptabilite & $0.29 \%$ & $0.29 \%$ & $0.29 \%$ \\
\hline collectif & $3.04 \%$ & $3.12 \%$ & $3.09 \%$ & autonomie & $0.59 \%$ & $0.47 \%$ & $0.51 \%$ \\
\hline mutualisation & $0.10 \%$ & $0.71 \%$ & $0.48 \%$ & automerric & $0.20 \%$ & $0.41 \%$ & $0.33 \%$ \\
\hline echange & $0.49 \%$ & $0.35 \%$ & $0.40 \%$ & politique & $0.88 \%$ & $0.12 \%$ & $0.40 \%$ \\
\hline vivre_ensemble & $0.10 \%$ & $0.47 \%$ & $0.33 \%$ & & & & \\
\hline collectif & $0.39 \%$ & $0.29 \%$ & $0.33 \%$ & & & & \\
\hline coopcration & $0.00 \%$ & $0.41 \%$ & $0.26 \%$ & & & & \\
\hline
\end{tabular}

Figures 1a,b,c : Pourcentage d'occurrences des termes lors de l'activité 1 (1 020 termes), de l'activité 3 (1 700 termes) et au total des activités 1 et 3 regroupés en 21 catégories.

Figure $1 a$ : catégories 1 à 4 ; Figure $1 b$ : catégories 5 à 10 ; Figure $1 c$ : catégories 11 à 21 . Seuls les termes ayant eu au moins sept occurrences au total des activités 1 et 3 sont indiqués dans cette table.

Les corpus sont ensuite traités au moyen du logiciel IRaMuTeQ dans sa version 0.7 alpha 2 qui selon Loubère et Ratinaud (2014) « propose un ensemble de traitements et d'outils pour l'aide à la description et à l'analyse de matrices de type individus/ caractères ». Nous avons réalisé deux types d'analyses: (1) une analyse prototypique permettant de révéler l'organisation structurale de la représentation sociale et (2) une analyse de similitudes permettant d'identifier les liens qu'entretiennent les éléments entre eux.

\section{Analyse prototypique}

Dans une population donnée, on obtient un corpus d'éléments - le contenu de la représentation - et deux indicateurs quantitatifs pour chaque élément évoqué : (1) sa fréquence d'apparition (nombre de citations) et (2) le degré moyen d'importance que le sujet lui accorde (rang d'apparition dans le classement des mots à savoir de 1 à 3 pour l'activité 1 et de 1 à 5 pour l'activité 3). L'analyse prototypique est une méthode descriptive qui consiste à croiser la fréquence d'apparition et le rang moyen d'importance de chaque élément pour obtenir une première identification de l'organisation structurale de la représentation sociale par la distinction de quatre zones (Abric, 2005 ; Figure 2) : (1) le noyau central, (2) les éléments contrastés, (3) la première périphérie et (4) la seconde périphérie. 


\begin{tabular}{|c|c|c|}
\hline & Rang inférieur ou égal au rang moyen & Rang supérieur au rang moyen \\
\hline $\begin{array}{l}\text { Fréquence } \\
\text { supérieure } \\
\text { ou égale } \\
\text { à la fréquence } \\
\text { moyenne }\end{array}$ & \begin{tabular}{|c|} 
Noyau central \\
Éléments qui sont considérés comme les plus \\
importants de la représentation car les plus \\
fréquents (fréquence élevée) et classés dans \\
les premiers (rang faible).
\end{tabular} & $\begin{array}{l}\text { Première périphérie } \\
\text { Zone de changement potentiel correspondant } \\
\text { aux éléments complémentaires du noyau } \\
\text { central (fréquence élevée) mais de moindre } \\
\text { importance pour le locuteur (rang élevé). }\end{array}$ \\
\hline $\begin{array}{l}\text { Fréquence } \\
\text { inférieure } \\
\text { à la fréquence } \\
\text { moyenne }\end{array}$ & $\begin{array}{c}\text { Eléments contrastés } \\
\text { Zone de changement potentiel correspondant } \\
\text { aux éléments proposés par un nombre limité } \\
\text { de personnes (fréquence faible) mais } \\
\text { néanmoins considérés comme importants par } \\
\text { le locuteur (rang faible). }\end{array}$ & $\begin{array}{l}\text { Seconde périphérie } \\
\text { Éléments peu évoqués (fréquence faible) et } \\
\text { peu importants pour le locuteur (rang élevé) } \\
\text { et donc considérés comme les moins } \\
\text { importants de la représentation. }\end{array}$ \\
\hline
\end{tabular}

Figure 2 : Lecture de l'analyse prototypique (adapté d'Abric, 2005).

\section{Analyse de similitudes}

L'analyse de similitudes définie par Flament (1962) permet d'identifier les liens qu'entretiennent les éléments entre eux. Plus précisément, elle révèle les relations qui sont les plus significatives entre les éléments analysés par le calcul des cooccurrences. En formalisant ainsi les relations entre éléments, l'analyse de similitudes apporte des informations supplémentaires quant à la structure des réponses et aux connexions entre les éléments. Dans le cas présent, l'algorithme de Fruchterman Reingold (1991) a été utilisé pour optimiser l'affichage du graphique d'analyse de similitudes et visualiser ainsi les mots les plus « centraux » servant d'intermédiaires aux autres mots.

La détection des communautés a quant à elle été réalisée en utilisant la méthode dite des bordures entre communautés (" edge betweenness community») développée par Girvan et Newman (2002) qui définit le concept de bordure comme l'ensemble des nœuds ayant au moins un lien vers une autre communauté. Plus concrètement, cette méthode permet de regrouper les termes fortement cooccurrents au sein d'une même communauté en divisant de façon itérative les nœuds d'un réseau en une hiérarchie de communautés imbriquées.

\section{Résultats}

31 L'analyse des résultats repose sur l'exploitation des tableaux prototypiques et des analyses de similitudes obtenus lors de l'activité 1 puis lors de l'activité 3 afin (1) d'identifier les contenus des représentations, (2) de dessiner les contours de leurs structures et (3) de comprendre les liens tissés entre elles au travers de la mise en évidence des communautés de termes.

\section{Analyses prototypique et de similitudes lors de l'activité 1}

\section{Analyse prototypique de l'activité 1}

Pour l'ensemble de la population, le noyau central de l'activité 1 (Figure 3) correspond aux rangs inférieurs ou égaux à 2,0 et aux fréquences supérieures ou égales à $4,8 \%$. Il 
est constitué de six termes qui, classés par ordre décroissant de fréquence, sont les suivants : technique, environnement, changement, durable, savoir et agriculture.

La première périphérie du noyau central (fréquence $\geq 4,8 \%$ et rang $>2,0$ ) montre qu'à l'issue de l'activité 1 , « produire autrement » est pour les enseignants.es en lien direct avec les notions de territoire et d'alimentation. Les éléments contrastés (fréquence $<4,8 \%$ et rang $\leq 2,0$ ) sont au nombre de quatre: agroécologie, éducation, valeur et alternatif. Enfin, en seconde périphérie nous retrouvons les neufs termes restants, les moins cités et les moins importants pour les enseignants.es (fréquence $<4,8 \%$ et rang $>2,0$ ).

\begin{tabular}{|c|c|c|c|c|c|}
\hline $\begin{array}{r}\text { Ran } \\
\text { Noyau }\end{array}$ & $\begin{array}{l}2 \\
\text { ntral }\end{array}$ & & $\begin{aligned} & 2 \\
& \text { Premièr }\end{aligned}$ & $\begin{array}{l}\text { ang } \\
\text { ériphé }\end{array}$ & \\
\hline & $\%$ & Rang & & $\%$ & Rang \\
\hline technique & $12.6 \%$ & 2.0 & territoire & $5.7 \%$ & 2.1 \\
\hline environnement & $12.3 \%$ & 2.0 & alimentation & $5.4 \%$ & 2.2 \\
\hline changement & $10.5 \%$ & 2.0 & & & \\
\hline durable & $9.3 \%$ & 1.7 & & & \\
\hline savoir & $5.7 \%$ & 1.9 & & & \\
\hline agriculture & $5.6 \%$ & 1.8 & & & \\
\hline Eléments & ntrasté: & & Seconde & riphér & \\
\hline & $\%$ & Rang & & $\%$ & Rang \\
\hline agroecologie & $4.3 \%$ & 1.7 & economie & $3.9 \%$ & 2.4 \\
\hline education & $4.3 \%$ & 1.9 & social & $3.6 \%$ & 2.1 \\
\hline valeur & $4.1 \%$ & 2.0 & collectif & $3.0 \%$ & 2.3 \\
\hline alternatif & $1.2 \%$ & 1.9 & avenir & $2.2 \%$ & 2.2 \\
\hline & & & diversite & $1.9 \%$ & 2.1 \\
\hline & & & bien_etre & $1.8 \%$ & 2.3 \\
\hline & & & adaptation & $1.2 \%$ & 2.1 \\
\hline & & & politique & $0.9 \%$ & 2.6 \\
\hline & & & autonomie & $0.6 \%$ & 2.3 \\
\hline
\end{tabular}

Figure 3 : Analyse prototypique de la représentation individuelle du " produire autrement » obtenue lors de l'activité 1 pour l'ensemble de la population $(n=340)$.

L'analyse prototypique de chacun des six sous-ensembles de la population (Figures $4 \mathrm{abc}$ ) montre, comme celle de la population totale, que les termes environnement et durable sont systématiquement présents dans le noyau central. Il en va de même du terme changement sauf pour le groupe «non technique » qui le place dans la première périphérie. Le terme savoir est également présent dans le noyau central des sous-ensembles de la population sauf pour les groupes « homme » et « technique » qui le situent dans la première périphérie. Le terme technique se retrouve quant à lui dans le noyau central pour les groupes "femme ", "expérimenté » et " non technique ", mais en première périphérie pour les autres alors que l'inverse est observé pour la notion de territoire. On remarque également que les groupes "novice » et "technique " positionnent le terme agriculture dans les éléments contrastés et que les groupes "femme» et "non technique» positionnent le terme alimentation en seconde périphérie. Soulignons que le terme éducation se situe dans le noyau central des groupes " expérimenté » et "non technique », et le terme agroécologie dans celui des groupes «technique » et "expérimenté ». Enfin dans le cas du groupe «non technique » on notera l'absence d'éléments contrastés. 
Figure 4a

\begin{tabular}{|c|c|c|c|c|c|}
\hline \multicolumn{6}{|c|}{ Technique } \\
\hline \multicolumn{2}{|c|}{ Noyau central } & \multicolumn{4}{|c|}{ Première périphérie } \\
\hline & $\%$ & Rang & & $\%$ & Rang \\
\hline environnement & $13.1 \%$ & 2.0 & technique & $15.4 \%$ & 2.2 \\
\hline durable & $11.1 \%$ & 1.8 & alimentation & $7.1 \%$ & 2.2 \\
\hline changement & $6.8 \%$ & 2.0 & savoir & $5.1 \%$ & 2.4 \\
\hline agroecologie & $6.8 \%$ & 1.5 & & & \\
\hline lerritoire & $5.4 \%$ & 1.8 & & & \\
\hline \multicolumn{3}{|c|}{ Eléments contrastés } & \multicolumn{3}{|c|}{ Seconde périphérie } \\
\hline & $\%$ & Rang & & $\%$ & Rang \\
\hline valcur & $4.3 \%$ & 1.8 & avenir & $2.0 \%$ & 2.1 \\
\hline collectif & $4.0 \%$ & 2.0 & education & $1.7 \%$ & 2.2 \\
\hline economie & $3.7 \%$ & 1.9 & autonomie & $1.4 \%$ & 2.2 \\
\hline agriculture & $3.4 \%$ & 1.8 & adaptation & $1.1 \%$ & 2.2 \\
\hline social & $2.6 \%$ & 2.0 & politique & $0.9 \%$ & 2.3 \\
\hline alternatif & $1.4 \%$ & 1.8 & & & \\
\hline bien_ctre & $1.4 \%$ & 2.0 & & & \\
\hline diversite & $1.1 \%$ & 1.8 & & & \\
\hline
\end{tabular}

\begin{tabular}{|c|c|c|c|c|c|}
\hline \multicolumn{6}{|c|}{ Non technique } \\
\hline \multicolumn{3}{|c|}{ Noyau central } & \multicolumn{3}{|c|}{ Première périphérie } \\
\hline & $\%$ & Rang & & $\%$ & Rang \\
\hline environnement & $11.8 \%$ & 1.9 & changement & $12.4 \%$ & 2.0 \\
\hline lechnique & $11.2 \%$ & 1.9 & lerritoire & $5.8 \%$ & 2.2 \\
\hline durable & $8.4 \%$ & 1.6 & & & \\
\hline agriculture & $6.7 \%$ & 1.9 & & & \\
\hline savoir & $6.0 \%$ & 1.7 & & & \\
\hline education & $5.7 \%$ & 1.9 & & & \\
\hline \multicolumn{3}{|c|}{ Eléments contrastés } & \multicolumn{3}{|c|}{ Seconde périphérie } \\
\hline & $\%$ & Rang & & $\%$ & Rang \\
\hline & & & alimentation & $4.5 \%$ & 2.2 \\
\hline & & & social & $4.2 \%$ & 2.1 \\
\hline & & & economie & $4.0 \%$ & 2.6 \\
\hline & & & valcur & $4.0 \%$ & 2.1 \\
\hline & & & agroecologie & $3.0 \%$ & 2.0 \\
\hline & & & collectif & $2.5 \%$ & 2.5 \\
\hline & & & avenir & $2.2 \%$ & 2.3 \\
\hline & & & diversile & $2.2 \%$ & 2.1 \\
\hline & & & bien_etre & $1.9 \%$ & 2.4 \\
\hline & & & adaptation & $1.2 \%$ & 2.0 \\
\hline & & & altemalit & $1.0 \%$ & 2.0 \\
\hline & & & politique & $0.9 \%$ & 2.7 \\
\hline
\end{tabular}

\section{Figure 4b}

\begin{tabular}{|c|c|c|c|c|c|}
\hline \multicolumn{6}{|c|}{ Novice } \\
\hline \multicolumn{2}{|c|}{ Noyau central } & \multicolumn{4}{|c|}{ Première périphérie } \\
\hline & $\%$ & Rang & & $\%$ & Rang \\
\hline environnement & $13.1 \%$ & 1.9 & technique & $9.1 \%$ & 2.2 \\
\hline durable & $11.4 \%$ & 1.7 & alimentation & $5.7 \%$ & 2.2 \\
\hline changement & $10.8 \%$ & 1.9 & & & \\
\hline savoir & $7.4 \%$ & 2.0 & & & \\
\hline territoire & $7.1 \%$ & 1.8 & & & \\
\hline \multicolumn{3}{|c|}{ Eléments contrastés } & \multicolumn{3}{|c|}{ Seconde périphérie } \\
\hline & $\%$ & Rang & & $\%$ & Rang \\
\hline agriculture & $4.6 \%$ & 1.9 & valeur & $4.6 \%$ & 2.0 \\
\hline agroecologie & $3.4 \%$ & 1.7 & social & $3.7 \%$ & 2.4 \\
\hline cducation & $2.3 \%$ & 1.9 & cconomic & $3.4 \%$ & 2.3 \\
\hline bien_etre & $2.0 \%$ & 1.7 & alvenir & $2.3 \%$ & 2.6 \\
\hline collectif & $2.0 \%$ & 2.0 & adaptation & $2.3 \%$ & 2.1 \\
\hline alternatif & $1.1 \%$ & 1.2 & politique & $1.4 \%$ & 2.6 \\
\hline diversite & $1.1 \%$ & 2.0 & autonomic & $1.1 \%$ & 2.2 \\
\hline
\end{tabular}

\begin{tabular}{|c|c|c|c|c|c|}
\hline \multicolumn{6}{|c|}{ Expérimenté } \\
\hline \multicolumn{3}{|c|}{ Noyau central } & \multicolumn{3}{|c|}{ Première périphérie } \\
\hline & $\%$ & Rang & & $\%$ & Rang \\
\hline technique & $14.5 \%$ & 2.0 & alimentation & $5.2 \%$ & 2.2 \\
\hline environnement & $11.8 \%$ & 2.0 & territoire & $4.9 \%$ & 2.3 \\
\hline changement & $10.3 \%$ & 2.0 & & & \\
\hline durable & $8.2 \%$ & 1.6 & & & \\
\hline agriculture & $6.1 \%$ & 1.8 & & & \\
\hline education & $5.4 \%$ & 1.9 & & & \\
\hline agroccologic & $4.8 \%$ & 1.7 & & & \\
\hline savoir & $4.8 \%$ & 1.9 & & & \\
\hline \multicolumn{3}{|c|}{ Eléments contrastés } & \multicolumn{3}{|c|}{ Seconde périphérie } \\
\hline & $\%$ & Rang & & $\%$ & Rang \\
\hline valeur & $3.9 \%$ & 1.9 & economie & $4.2 \%$ & 2.4 \\
\hline social & $3.6 \%$ & 2.0 & collectif & $3.6 \%$ & 2.3 \\
\hline avenir & $2.1 \%$ & 2.0 & diversite & $2.2 \%$ & 2.1 \\
\hline \multirow{4}{*}{ adaptation } & $0.6 \%$ & 2.0 & bien_etre & $1.6 \%$ & 2.6 \\
\hline & & & alternatif & $1.2 \%$ & 2.2 \\
\hline & & & politique & $0.6 \%$ & 2.5 \\
\hline & & & autonomic & $0.3 \%$ & 2.5 \\
\hline
\end{tabular}

Figure 4c

\begin{tabular}{|c|c|c|c|c|c|}
\hline & & FemI & me & & \\
\hline Noyau & ntral & & Premièr & Ériphér & \\
\hline & $\%$ & Rang & & $\%$ & Rang \\
\hline changement & $13.0 \%$ & 2.0 & territoire & $5.0 \%$ & 2.2 \\
\hline environnement & $12.9 \%$ & 2.0 & & & \\
\hline technique & $10.5 \%$ & 2.0 & & & \\
\hline durable & $8.7 \%$ & 1.7 & & & \\
\hline savoir & $6.3 \%$ & 1.9 & & & \\
\hline agriculture & $6.0 \%$ & 1.8 & & & \\
\hline Eléments & ntrastés & & Seconde & riphéri & \\
\hline & $\%$ & Rang & & $\%$ & Rang \\
\hline education & $4.8 \%$ & 1.8 & alimentation & $4.5 \%$ & 2.1 \\
\hline agroccologic & $4.5 \%$ & 1.8 & cconomic & $4.0 \%$ & 2.5 \\
\hline social & $3.3 \%$ & 1.9 & valeur & $3.8 \%$ & 2.1 \\
\hline diversite & $2.0 \%$ & 1.9 & collectil & $3.0 \%$ & 2.3 \\
\hline alternatif & $1.2 \%$ & 2.0 & avenir & $2.2 \%$ & 2.1 \\
\hline & & & bien_etre & $2.0 \%$ & 2.4 \\
\hline & & & adaptation & $1.3 \%$ & 2.2 \\
\hline & & & politique & $0.8 \%$ & 2.8 \\
\hline
\end{tabular}

\begin{tabular}{|c|c|c|c|c|c|}
\hline \multicolumn{6}{|c|}{ Homme } \\
\hline \multicolumn{2}{|c|}{ Noyau central } & \multicolumn{4}{|c|}{ Première périphérie } \\
\hline & $\%$ & Rang & & $\%$ & Rang \\
\hline environnement & $11.4 \%$ & 1.9 & technique & $15.7 \%$ & 2.1 \\
\hline durablc & $10.2 \%$ & 1.6 & alimentation & $6.7 \%$ & 2.2 \\
\hline changement & $6.9 \%$ & 1.9 & savoir & $4.8 \%$ & 2.1 \\
\hline territoire & $6.7 \%$ & 2.0 & & & \\
\hline agriculture & $5.0 \%$ & 2.0 & & & \\
\hline \multicolumn{3}{|c|}{ Eléments contrastés } & \multicolumn{3}{|c|}{ Seconde périphérie } \\
\hline & $\%$ & Rang & & $\%$ & Rang \\
\hline valeur & $4.5 \%$ & 1.8 & social & $4.0 \%$ & 2.4 \\
\hline agroccologic & $4.0 \%$ & 1.6 & cconomic & $3.8 \%$ & 2.2 \\
\hline bien_etre & $1.4 \%$ & 2.0 & education & $3.6 \%$ & 2.2 \\
\hline alternatif & $1.2 \%$ & 1.8 & collectil & $3.1 \%$ & 2.2 \\
\hline \multirow[t]{4}{*}{ adaptation } & $1.0 \%$ & 1.8 & avenir & $2.1 \%$ & 2.4 \\
\hline & & & diversite & $1.7 \%$ & 2.3 \\
\hline & & & autonomie & $1.2 \%$ & 2.4 \\
\hline & & & politique & $1.0 \%$ & 2.2 \\
\hline
\end{tabular}

Figure 4a,b,c: Analyse prototypique de la représentation individuelle du « produire autrement » obtenue lors de l'activité 1 pour les six sous-ensembles de la population.

Figure 4a : Technique $(n=117)$ et Non technique $(n=223)$; Figure $4 b$ : Novice $(n=117)$ et Expérimenté $(n=223)$; Figure $4 c$ : Femme $(n=200)$ et Homme $(n=140)$. 


\section{Analyse de similitudes lors de l'activité 1}

(Figure 5) montre une organisation en quatre communautés structurées par ordre décroissant de fréquence d'occurrences autour des termes centraux : environnement (31,1\%), changement (24,2\%), technique (23,5\%) et durable (21,2\%).

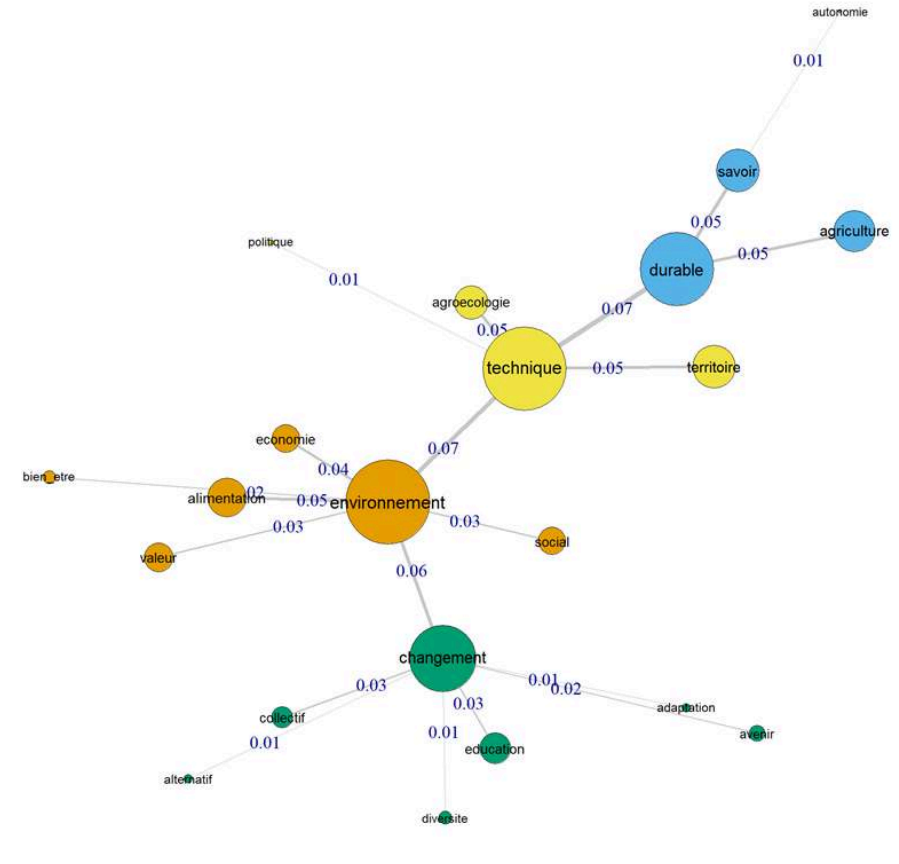

Figure 5 : Analyse de similitudes par l'algorithme de Fruchterman Reingold (1991) de la représentation individuelle du " produire autrement » obtenue lors de l'activité 1 pour l'ensemble de la population $(n=340)$.

La taille des disques est proportionnelle au nombre d'occurrences. Un ensemble de disques de même couleur forme une communauté identifiée par la méthode des bordures entre communautés (« edge betweenness community ") d'après Girvan et Newman (2002). Les nombres bleus sur les droites reliant deux disques correspondent au taux de cooccurrence.

Les résultats montrent que les représentations ne sont pas homogènes pour les six sous-ensembles de la population (Figures $6 \mathrm{abc}$ ) avec plus ou moins de communautés et des différences dans la nature des termes constitutifs de ces dernières. Ainsi, la représentation $\mathrm{du}$ groupe "technique " s'articule en quatre communautés (environnement, technique, durable et alimentation) alors que celle du groupe «non technique » est constituée de cinq communautés (environnement, changement, technique, durable et diversité). Pour la population "novice», on observe quatre communautés (technique, durable, savoir et alimentation) contre seulement deux pour le groupe " expérimenté » (technique et environnement). Le groupe "femme" est quant à lui structuré en quatre communautés (changement, durable, environnement et technique) contre trois pour le groupe « homme» (technique, environnement et durable). 
Figure $6 \mathrm{a}$

Technique

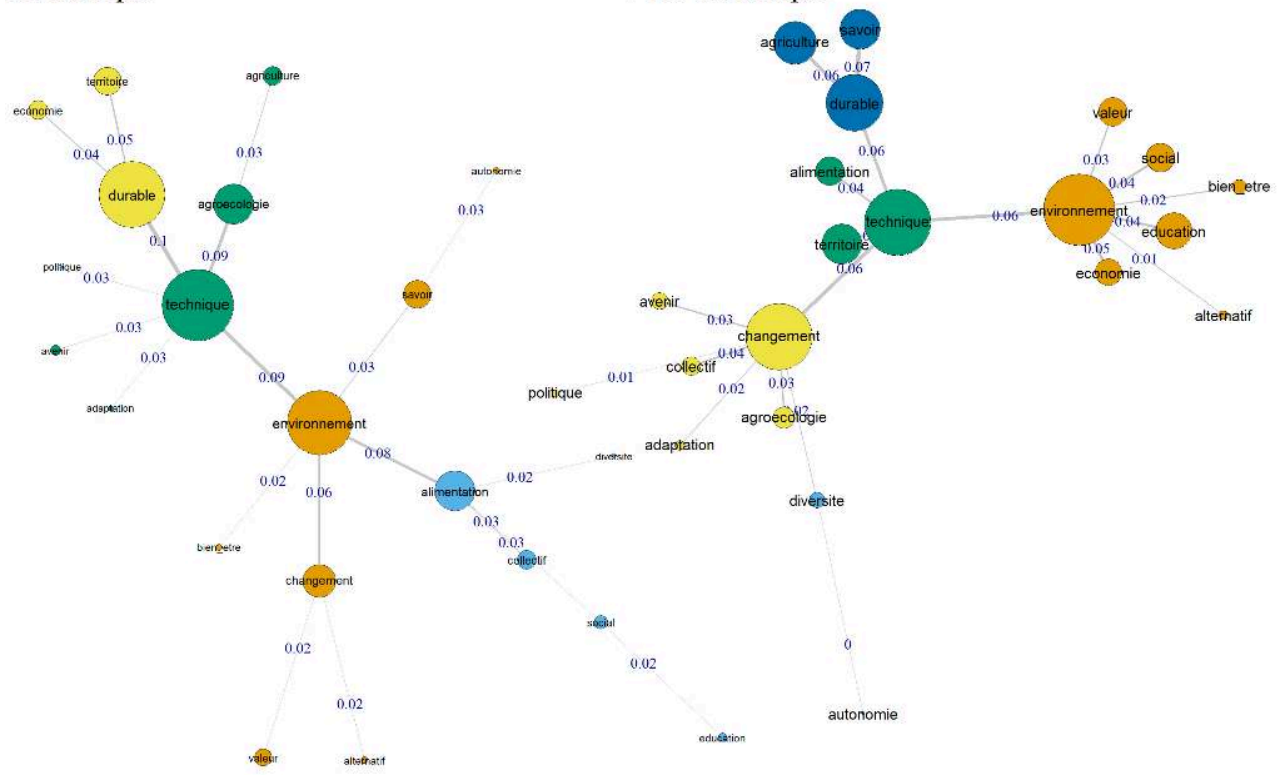

Figure $6 \mathrm{~b}$

Novice

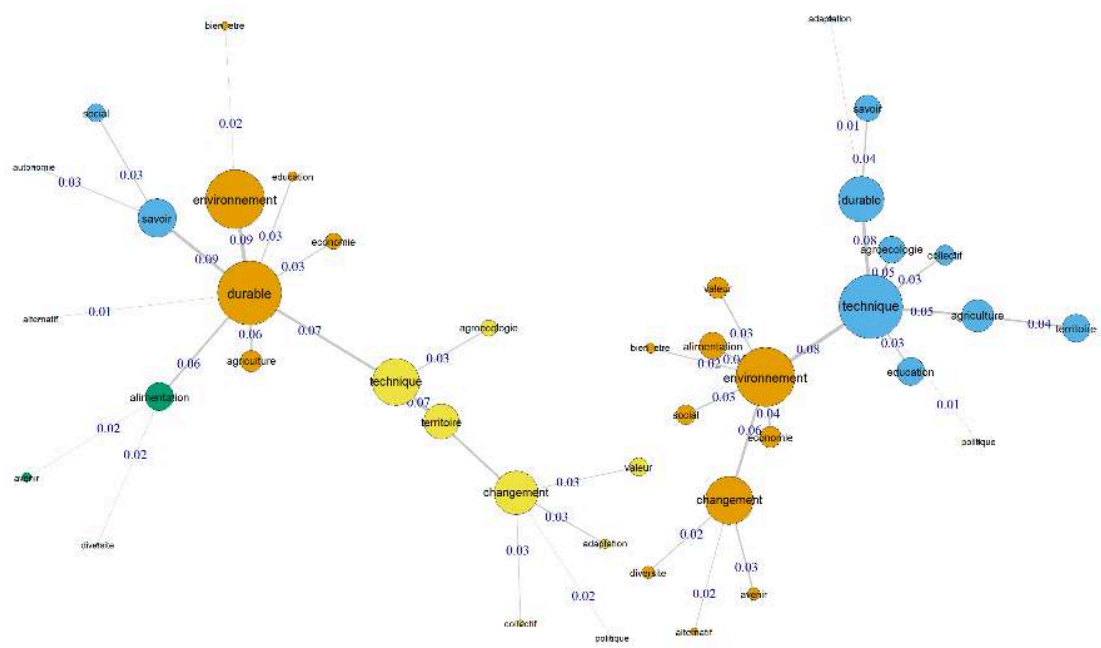

Expérimenté 
Figure 6c

Femme
Homme

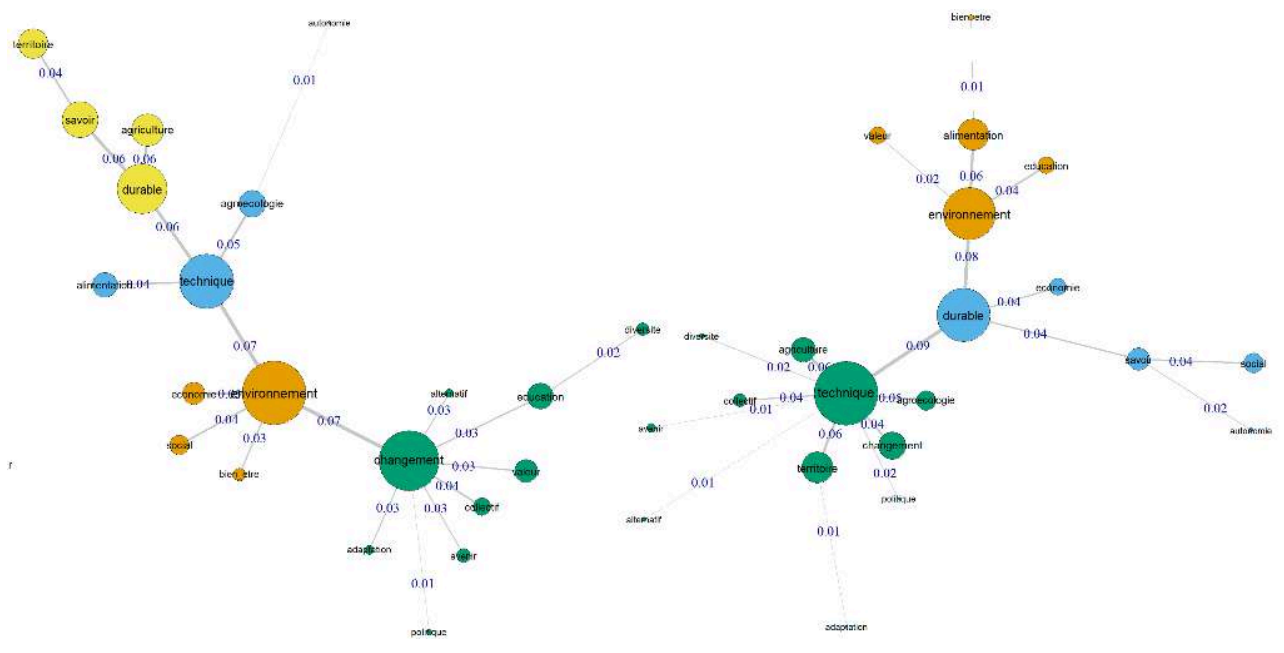

Figure 6 : Analyse de similitudes par l'algorithme de Fruchterman Reingold (1991) de la représentation individuelle du « produire autrement » obtenue lors de l'activité 1 pour les six sousensembles de la population.

Figure 6a: Technique $(n=117)$ et Non technique $(n=223)$; Figure $6 b$ : Novice $(n=117)$ et Expérimenté $(n=223)$; Figure $6 c$ : Femme $(n=200)$ et Homme $(n=140)$. La taille des disques est proportionnelle au nombre d'occurrences. Un ensemble de disques de même couleur forme une communauté identifiée par la méthode des bordures entre communautés (" edge betweenness community ») d'après Girvan et Newman (2002). Les nombres bleus sur les droites reliant deux disques correspondent au taux de cooccurrence.

\section{Analyses prototypique et de similitude lors de l'activité 3}

\section{Analyse prototypique lors de l'activité 3}

Pour l'ensemble de la population, le noyau central de l'activité 3 (Figure 7) correspond aux rangs inférieurs ou égaux à 3,0 et aux fréquences supérieures ou égales à $4,8 \%$. Il est constitué de quatre termes qui sont par ordre décroissant de fréquence: changement, durable, environnement et technique.

La première périphérie du noyau central (fréquence $\geq 4,8 \%$ et rang $>3,0$ ) est quant à elle constituée par éducation, territoire, valeur et bien-être. Les éléments contrastés (fréquence $<4,8 \%$ et rang $\leq 3,0$ ) sont au nombre de cinq: agroécologie, alternatif, agriculture, diversité et plus marginalement, politique. En seconde périphérie (fréquence $<4,8 \%$ et rang $>3,0$ ), nous retrouvons les huit termes restants qui sont les moins cités et les moins importants pour les enseignants.es. 


\begin{tabular}{|c|c|c|c|c|c|c|}
\hline & $\begin{array}{r}\text { Ran } \\
\text { Noyau }\end{array}$ & $\begin{array}{l}3 \\
\text { ntral }\end{array}$ & & $\begin{array}{r}3 \\
\text { Premièr }\end{array}$ & $\begin{array}{l}\text { ang } \\
\text { ériphér }\end{array}$ & \\
\hline & & $\%$ & Rang & & $\%$ & Rang \\
\hline & changement & $10.9 \%$ & 2.7 & education & $12.3 \%$ & 3.3 \\
\hline & durable & $9.8 \%$ & 2.5 & territoire & $7.9 \%$ & 3.1 \\
\hline & environnement & $9.1 \%$ & 2.9 & valeur & $7.1 \%$ & 3.2 \\
\hline & technique & $5.2 \%$ & 3.0 & bien_etre & $5.4 \%$ & 3.4 \\
\hline & Eléments & ntrastés & & Second & ériphéri & \\
\hline & & $\%$ & Rang & & $\%$ & Rang \\
\hline & agroecologie & $4.4 \%$ & 2.2 & alimentation & $4.3 \%$ & 3.4 \\
\hline & alternatif & $3.0 \%$ & 2.8 & savoir & $3.9 \%$ & 3.2 \\
\hline & agriculture & $1.9 \%$ & 2.1 & social & $3.7 \%$ & 3.4 \\
\hline & diversite & $1.1 \%$ & 2.9 & avenir & $3.6 \%$ & 3.2 \\
\hline & politique & $0.1 \%$ & 3.0 & collectif & $3.1 \%$ & 3.2 \\
\hline & & & & economie & $1.6 \%$ & 3.3 \\
\hline & & & & adaptation & $1.1 \%$ & 3.3 \\
\hline & & & & autonomie & $0.5 \%$ & 3.2 \\
\hline
\end{tabular}

Figure 7 : Analyse prototypique de la représentation individuelle du « produire autrement » obtenue lors de l'activité 3 pour l'ensemble de la population $(n=340)$.

Comme dans le cas de l'analyse de l'ensemble de la population, les termes changement et durable sont systématiquement présents dans le noyau central et le terme éducation, systématiquement présent dans la première périphérie et ce quel que soit le sousensemble de la population considéré (Figures $8 \mathrm{abc}$ ). Les termes environnement et technique sont également situés dans le noyau central des sous-ensembles de la population sauf pour le groupe «novice » qui place environnement dans la première périphérie et les groupes «novice » et «non technique » qui positionnent le terme technique dans les éléments contrastés. À l'inverse, les termes territoire et valeur sont situés en première périphérie des sous-ensembles de la population sauf pour les groupes «homme » et "novice " qui placent dans le noyau central respectivement territoire et valeur. Soulignons que le terme agroécologie se situe dans le noyau central des groupes «technique » et "expérimenté » et que le terme bien-être est dans la première périphérie pour les groupes "non technique ", " expérimenté » et "femme ». Enfin dans le cas du groupe "novice » la première périphérie intègre quatre autres termes (avenir, savoir, alimentation et social). 
Figure 8a

\begin{tabular}{|c|c|c|c|c|c|}
\hline \multicolumn{6}{|c|}{ Technique } \\
\hline \multicolumn{2}{|c|}{ Noyau central } & \multicolumn{4}{|c|}{ Première périphérie } \\
\hline & $\%$ & Rang & & $\%$ & Rang \\
\hline durable & $11.8 \%$ & 2.5 & cducation & $9.2 \%$ & 3.4 \\
\hline changement & $10.6 \%$ & 2.9 & valeur & $8.9 \%$ & 3.2 \\
\hline environnement & $9.7 \%$ & 3.0 & territoire & $7.7 \%$ & 3.2 \\
\hline technique & $6.7 \%$ & 2.9 & & & \\
\hline agroecologie & $6.5 \%$ & 2.3 & & & \\
\hline \multicolumn{3}{|c|}{ Eléments contrastés } & \multicolumn{3}{|c|}{ Seconde périphérie } \\
\hline & $\%$ & Rang & & $\%$ & Rang \\
\hline savoir & $3.9 \%$ & 3.0 & alimentation & $4.3 \%$ & 3.3 \\
\hline allematil & $3.1 \%$ & 2.7 & social & $3.6 \%$ & 3.5 \\
\hline collectif & $2.6 \%$ & 2.6 & bien_etre & $3.1 \%$ & 3.7 \\
\hline cconomic & $2.4 \%$ & 3.0 & avenir & $2.2 \%$ & 3.9 \\
\hline agriculture & $1.5 \%$ & 2.1 & adaptation & $0.7 \%$ & 3.2 \\
\hline autonomie & $1.0 \%$ & 3.0 & diversite & $0.5 \%$ & 4.0 \\
\hline
\end{tabular}

\begin{tabular}{|c|c|c|c|c|c|}
\hline \multicolumn{6}{|c|}{ Non technique } \\
\hline \multicolumn{3}{|c|}{ Noyau central } & \multicolumn{3}{|c|}{ Première périphérie } \\
\hline & $\%$ & Rang & & $\%$ & Rang \\
\hline changement & $11.1 \%$ & 2.7 & cducation & $13.9 \%$ & 3.3 \\
\hline environnement & $8.8 \%$ & 2.9 & Lerritoire & $8.1 \%$ & 3.1 \\
\hline durable & $8.8 \%$ & 2.5 & bien_etre & $6.6 \%$ & 3.4 \\
\hline & & & valcur & $6.1 \%$ & 3.2 \\
\hline \multicolumn{3}{|c|}{ Eléments contrastés } & \multicolumn{3}{|c|}{ Seconde périphérie } \\
\hline & $\%$ & Rang & & $\%$ & Rang \\
\hline technique & $4.4 \%$ & 3.0 & alimentation & $4.3 \%$ & 3.4 \\
\hline avenir & $4.3 \%$ & 3.0) & siavoir & $3.9 \%$ & 3.3 \\
\hline agroecologie & $3.3 \%$ & 2.0 & social & $3.8 \%$ & 3.4 \\
\hline alternatif & $3.0 \%$ & 2.9 & collectif & $3.4 \%$ & 3.5 \\
\hline agriculture & $2.1 \%$ & 2.1 & adaptation & $1.3 \%$ & 3.3 \\
\hline diversile & $1.3 \%$ & 2.7 & economie & $1.3 \%$ & 3.6 \\
\hline politique & $0.2 \%$ & 3.0 & autonomie & $0.2 \%$ & 4.0 \\
\hline
\end{tabular}

Figure 8b

\begin{tabular}{|c|c|c|c|c|c|}
\hline & & Novi & ice & & \\
\hline Noy: & ntral & & Première $p$ & riphér & \\
\hline & $\%$ & Rang & & $\%$ & Rang \\
\hline changement & $12.8 \%$ & 2.6 & environnement & $9.6 \%$ & 3.3 \\
\hline durable & $11.5 \%$ & 2.7 & territoirc & $7.5 \%$ & 3.1 \\
\hline valeur & $6.2 \%$ & 3.0 & education & $6.5 \%$ & 3.2 \\
\hline & & & avenir & $6.2 \%$ & 3.4 \\
\hline & & & savoir & $5.8 \%$ & 3.2 \\
\hline & & & alimentation & $5.3 \%$ & 3.2 \\
\hline & & & social & $5.3 \%$ & 3.2 \\
\hline Elémen & ntrastés & & $\overline{\text { Seconde } p}$ & íphéri & \\
\hline & $\%$ & Rang & & $\%$ & Rang \\
\hline technique & $4.8 \%$ & 2.9 & collectif & $3.4 \%$ & 3.3 \\
\hline agroecologie & $3.6 \%$ & 2.7 & adaptation & $1.9 \%$ & 3.4 \\
\hline bien_etre & $2.7 \%$ & 2.8 & autonomic & $0.9 \%$ & 3.6 \\
\hline agriculture & $2.4 \%$ & 1.9 & economie & $0.7 \%$ & 3.5 \\
\hline alternatir & $1.9 \%$ & 2.9 & & & \\
\hline diversite & $1.2 \%$ & 3.0 & & & \\
\hline
\end{tabular}

\begin{tabular}{|c|c|c|c|c|c|}
\hline \multicolumn{6}{|c|}{ Expérimenté } \\
\hline \multicolumn{3}{|c|}{ Noyau central } & \multicolumn{3}{|c|}{ Première périphérie } \\
\hline & $\%$ & Rang & & $\%$ & Rang \\
\hline changement & $10.0 \%$ & 2.8 & education & $15.3 \%$ & 3.3 \\
\hline durable & $9.0 \%$ & 2.4 & territoire & $8.2 \%$ & 3.2 \\
\hline environnement & $8.9 \%$ & 2.7 & valeur & $7.5 \%$ & 3.3 \\
\hline Lechnique & $5.4 \%$ & 3.0 & bien_etre & $6.8 \%$ & 3.6 \\
\hline agroecologie & $4.8 \%$ & 1.9 & & & \\
\hline \multicolumn{3}{|c|}{ Eléments contrastés } & \multicolumn{3}{|c|}{ Seconde périphérie } \\
\hline & $\%$ & Rang & & $\%$ & Rang \\
\hline altematif & $3.6 \%$ & 2.8 & alimentation & $3.8 \%$ & 3.5 \\
\hline avenir & $2.2 \%$ & 3.0 & collectif & $3.0 \%$ & 3.2 \\
\hline agriculturc & $1.6 \%$ & 2.3 & savoir & $2.9 \%$ & 3.1 \\
\hline diversite & $1.0 \%$ & 2.9 & social & $2.9 \%$ & 3.6 \\
\hline autonomie & $0.3 \%$ & 2.7 & economie & $2.2 \%$ & 3.3 \\
\hline politique & $0.2 \%$ & 3.0 & adaptation & $0.6 \%$ & 3.1 \\
\hline
\end{tabular}

Figure 8c

\begin{tabular}{|c|c|c|c|c|c|}
\hline \multicolumn{6}{|c|}{ Femme } \\
\hline \multicolumn{2}{|c|}{ Noyau central } & \multicolumn{4}{|c|}{ Première périphérie } \\
\hline & & Rang & & $\%$ & Rang \\
\hline changement & $11.3 \%$ & 2.8 & education & $13.7 \%$ & 3.1 \\
\hline environnement & $10.0 \%$ & 3.0 & territoire & $7.7 \%$ & 3.2 \\
\hline durable & $8.2 \%$ & 2.4 & valeur & $6.5 \%$ & 3.2 \\
\hline technique & $5.3 \%$ & 3.0 & bien_etre & $6.0 \%$ & 3.3 \\
\hline \multicolumn{3}{|c|}{ Eléments contrastés } & \multicolumn{3}{|c|}{ Seconde périphérie } \\
\hline & $\%$ & Rang & & $\%$ & Rang \\
\hline agroecologie & $4.2 \%$ & 2.0 & alimentation & $4.3 \%$ & 3.3 \\
\hline alternatif & $3.4 \%$ & 2.9 & savoir & $3.9 \%$ & 3.3 \\
\hline agriculture & $1.5 \%$ & 2.0 & avenir & $3.7 \%$ & 3.1 \\
\hline diversite & $1.1 \%$ & 2.7 & social & $3.3 \%$ & 3.5 \\
\hline adaptation & $0.9 \%$ & 3.0 & collectif & $2.7 \%$ & 3.4 \\
\hline \multirow[t]{2}{*}{ politicue } & $0.2 \%$ & 3.0 & economie & $1.6 \%$ & 3.0 \\
\hline & & & autonomie & $0.5 \%$ & 3.6 \\
\hline
\end{tabular}

\begin{tabular}{|c|c|c|c|c|c|}
\hline \multicolumn{6}{|c|}{ Homme } \\
\hline \multicolumn{2}{|c|}{ Noyau central } & \multicolumn{4}{|c|}{ Première périphérie } \\
\hline & $\%$ & Rang & & $\%$ & Rang \\
\hline durable & $12.1 \%$ & 2.6 & education & $10.3 \%$ & 3.6 \\
\hline changement. & $10.4 \%$ & 2.6 & valcur & $7.9 \%$ & 3.3 \\
\hline territoire & $8.3 \%$ & 3.0) & & & \\
\hline environnement & $7.9 \%$ & 2.8 & & & \\
\hline Lechnique & $5.0 \%$ & 2.9 & & & \\
\hline \multicolumn{3}{|c|}{ Eléments contrastés } & \multicolumn{3}{|c|}{ Seconde périphérie } \\
\hline & $\%$ & Rang & & $\%$ & Rang \\
\hline agroecologie & $4.7 \%$ & 2.4 & bien_etre & $4.6 \%$ & 3.6 \\
\hline savoir & $3.9 \%$ & 3.0 & alimentation & $4.3 \%$ & 3.5 \\
\hline altematif & $2.4 \%$ & 2.6 & social & $4.3 \%$ & 3.3 \\
\hline agriculture & $2.4 \%$ & 2.2 & collectif & $3.7 \%$ & 3.1 \\
\hline \multirow[t]{4}{*}{ autonomic } & $0.4 \%$ & 2.7 & avenir & $3.4 \%$ & 3.5 \\
\hline & & & economie & $1.7 \%$ & 3.2 \\
\hline & & & adaptation & $1.3 \%$ & 3.6 \\
\hline & & & diversite & $1.0 \%$ & 3.3 \\
\hline
\end{tabular}

Figures $\mathbf{8 a}, \mathrm{b}, \mathrm{c}$ : Analyse prototypique de la représentation individuelle du « produire autrement » obtenue lors de l'activité 3 pour les six sous-ensembles de la population.

Figure 8a: Technique $(n=117)$ et Non technique $(n=223)$; Figure 8b : Novice $(n=117)$ et Expérimenté $(n=223)$; Figure 8c : Femme $(n=200)$ et Homme $(n=140)$.

\section{Analyse de similitudes lors de l'activité 3}

L'analyse de l'arbre de similitudes de l'activité 3 pour l'ensemble de la population (Figure 9) montre une organisation autour de trois communautés structurées par ordre décroissant de fréquence d'occurrence autour des termes centraux : changement (45,6\% 
des citations), éducation (35,4\% des citations) et environnement (18,9\% des citations). À noter la place centrale occupée par la communauté éducation.

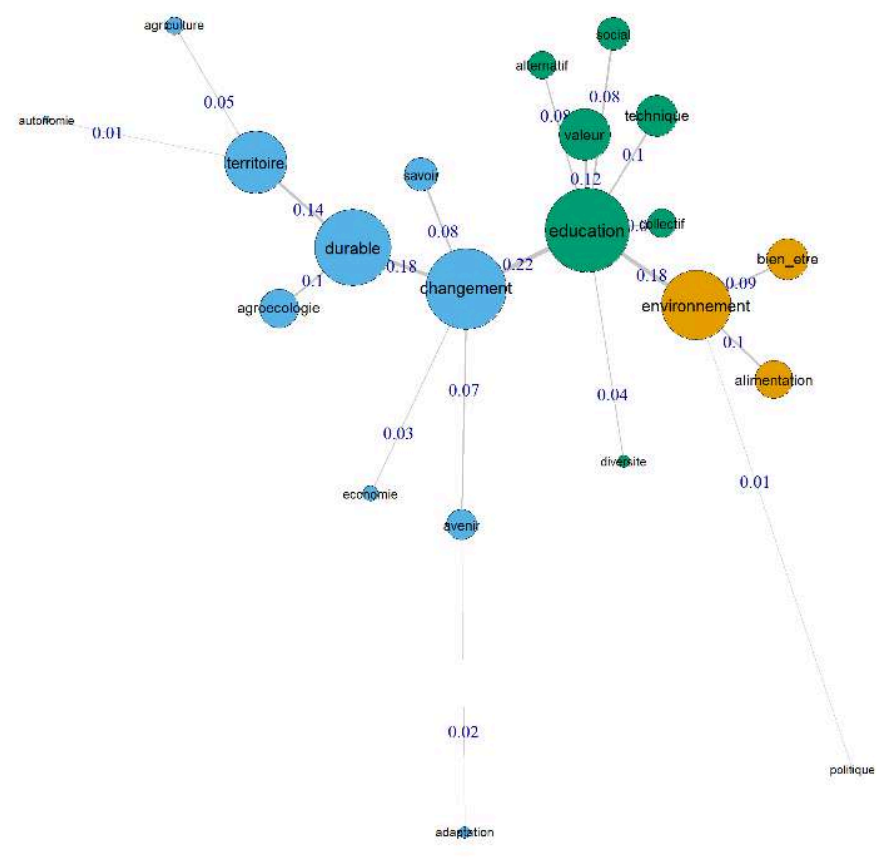

Figure 9 : Analyse de similitudes par l'algorithme de Fruchterman Reingold (1991) de la représentation individuelle du " produire autrement » obtenue lors de l'activité 3 pour l'ensemble de la population $(\mathrm{n}=340)$.

La taille des disques est proportionnelle au nombre d'occurrences. Un ensemble de disques de même couleur forme une communauté identifiée par la méthode des bordures entre communautés (" edge betweenness community ») d'après Girvan et Newman (2002). Les nombres bleus sur les droites reliant deux disques correspondent au taux de cooccurrence.

41 Comme précédemment, les représentations ne sont pas homogènes pour les six sousensembles de la population (Figures $10 \mathrm{abc}$ ). La représentation du groupe «technique » s'articule autour de quatre communautés (éducation, durable, environnement et changement) alors que celle du groupe "non technique" se construit autour de trois communautés (éducation, changement et durable). Pour la population "novice», deux communautés se dégagent (changement et durable) contre cinq pour le groupe « expérimenté » (éducation, changement, durable, environnement et bien-être). La représentation du groupe «femme » est quant à elle structurée en trois communautés (éducation, environnement et changement) tout comme celle du groupe "homme» (durable, éducation et changement). 
Figure 10a

Technique

Non technique

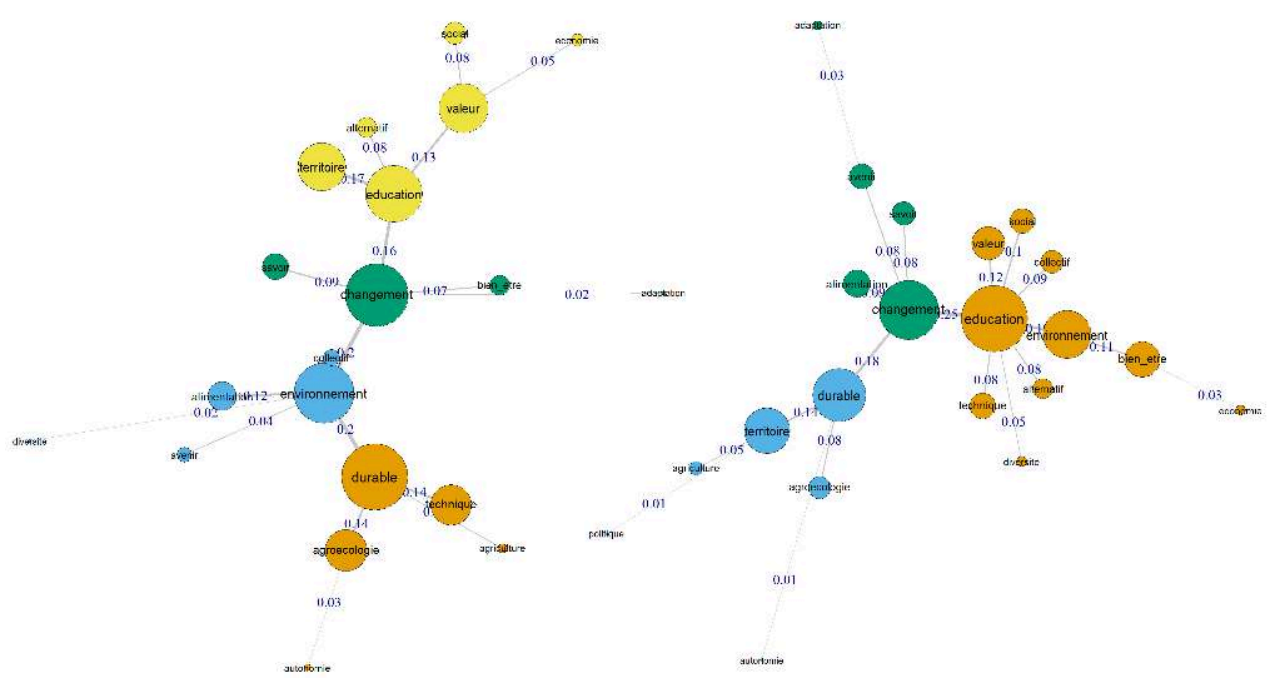

Figure $10 \mathrm{~b}$

Novice

Expérimenté

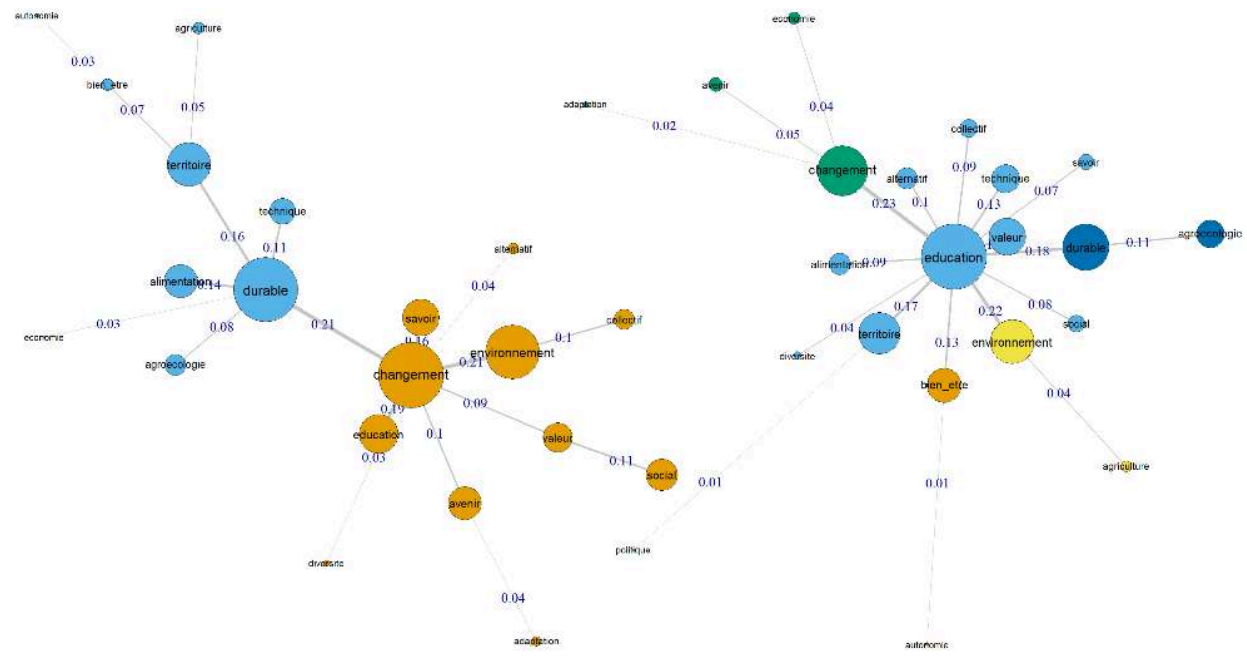


Figure 10c

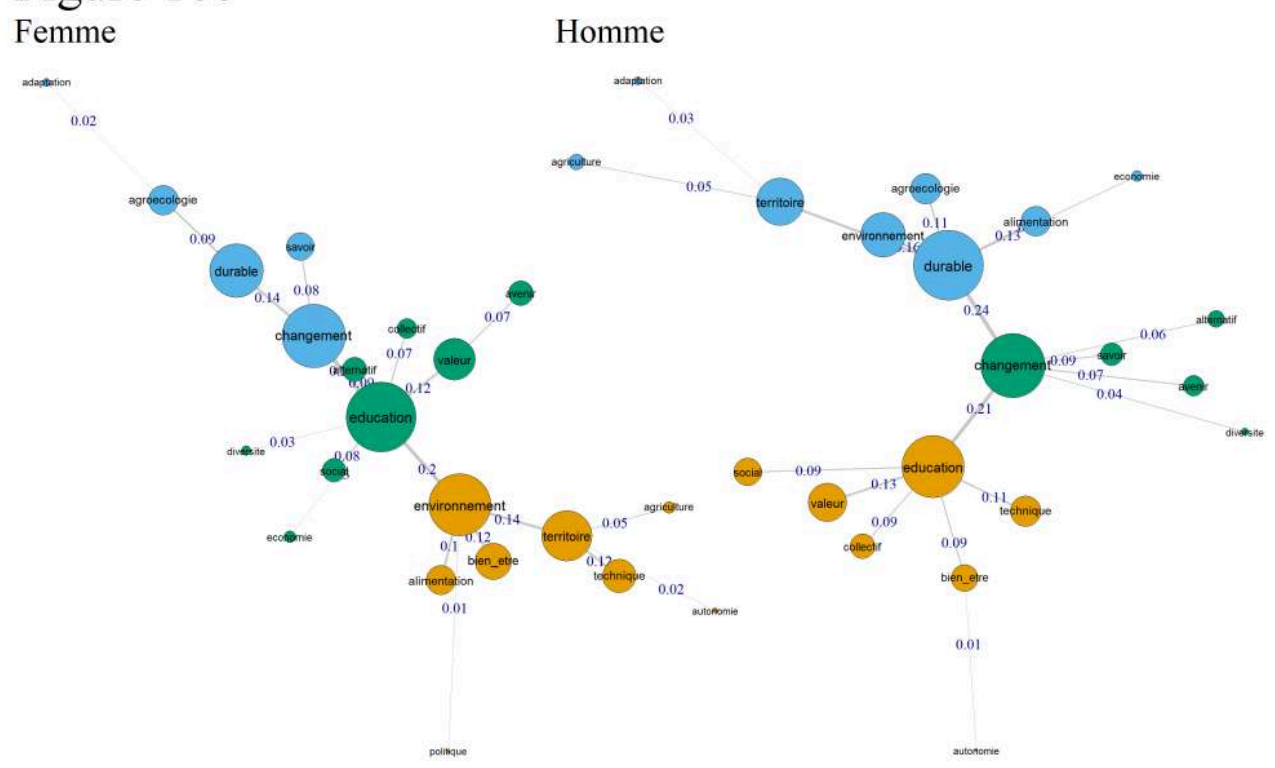

Figures 10a,b,c : Analyse de similitudes par l'algorithme de Fruchterman Reingold (1991) de la représentation individuelle du « produire autrement » obtenue lors de l'activité 3 pour les six sousensembles de la population.

Figure 10a: Technique $(n=117)$ et Non technique $(n=223)$; Figure 10b : Novice $(n=117)$ et Expérimenté $(n=223)$; Figure 10c : Femme $(n=200)$ et Homme $(n=140)$. La taille des disques est proportionnelle au nombre d'occurrences. Un ensemble de disques de même couleur forme une communauté identifiée par la méthode des bordures entre communautés (" edge betweenness community ») d'après Girvan et ewman (2002). Les nombres bleus sur les droites reliant deux disques correspondent au taux de cooccurrence.

\section{Discussion}

Nous souhaitions mettre à l'épreuve les deux hypothèses suivantes: (1) les représentations $d u$ "produire autrement " des enseignants.es sont a priori étroites et (2) les échanges entre enseignants.es de différentes disciplines et genres au cours de l'activité 2 peuvent induire une évolution dans leurs représentations.

\section{Des représentations a priori étroites du « produire autrement »}

43 L'analyse lexicale faite à l'issue de la première activité montre que le champ représentationnel est largement dominé par le terme technique même si celui-ci est associé à e des pratiques plus respectueuses de l'environnement qui est la deuxième référence la plus citée. Cela suggère que la représentation des enseignants.es reste avant tout cantonnée au pilier environnemental du développement durable sans forte référence aux deux autres (social et économie) qui n'apparaissent qu'en seconde périphérie. Toutefois, on peut penser que les enseignants.es ont implicitement inclus ces deux autres dimensions à travers le terme durable qui intervient en quatrième position.

Les enseignants.es mettent également en exergue la nécessité de changement des pratiques agricoles en évoquant les termes changement et agriculture qui font écho à l'acquisition et à la transmission du savoir qui apparaît en cinquième position. À noter 
que les éléments de la première périphérie, qui jouent un rôle important dans la significativité de la représentation, suggèrent à la fois une contextualisation du changement avec le terme territoire et l'expression du lien entre le champ et l'assiette avec l'évocation du terme alimentation.

À la lumière des résultats, il apparaît que les enseignants.es partagent au départ une certaine vision du "produire autrement ». En effet, la majorité des termes du noyau central et de la première périphérie (technique, environnement, changement, durable, savoir et territoire) sont communs à tous les sous-ensembles de la population. Toutefois, l'importance donnée à ces termes est différente et on observe également des notions spécifiques associées aux différents sous-ensembles de la population.

46 À titre d'exemple, le terme technique apparaît dans le noyau central du groupe «non technique » suggérant que la notion de «produire autrement » relève pour eux avant tout d'un changement dans la façon de produire faisant écho notamment au référentiel du baccalauréat professionnel «conduite et gestion de l'exploitation agricole » qui rappelle que «la transition agro-écologique constitue le nouveau modus operandi de la conduite et de l'accompagnement du changement dans les exploitations agricoles ». En extrapolant, on peut se demander si cette vision ne traduit pas le fait qu'ils se sentent pour partie exclus de cette injonction de "produire autrement ». Toutefois, la présence du terme éducation dans le noyau central, semble être une façon de justifier leur place dans le plan EPA2 qui « dépasse le domaine de la production agricole pour aborder celui de la transformation et des services ».

47 A contrario, les enseignants.es «technique " positionnent le terme technique dans la première périphérie lui préférant la notion d'agroécologie. Cela tend à suggérer que pour ces derniers, la notion " produire autrement » ne se limite pas à une vision techniciste mais recouvre une acception beaucoup plus large en lien avec celle d'agroécologie devenue plus familière pour eux en raison du projet agro-écologique pour la France lancé en 2014. De même, la population "expérimenté " fait apparaître un noyau dur plus complexe avec pas moins de huit termes dont technique, agroécologie et éducation, ce qui pourrait être l'expression de leur expérience professionnelle plus importante, suggérant qu'au-delà de faire évoluer les pratiques agricoles, l'enseignement poursuit aussi une visée éducative plus large visant à engager les élèves dans un positionnement réflexif et critique vis-à-vis des idéologies et des normes sociales (Sauvé, 1999 ; Girault et coll., 2007 ; Lange, 2015).

Ces différents éléments confirment donc l'hypothèse que nous avions formulée à savoir que les représentations individuelles du " produire autrement » des enseignants.es sont a priori étroites. Toutefois, la méthodologie employée se limite à quantifier l'importance donnée aux différents termes ; le « sens » qui leur est accordé reste discutable.

\section{Évolution des représentations permise par les échanges entre pairs}

49 À l'issue de l'activité 3, nous constatons que l'ordre des termes du noyau central est presque totalement inversé puisque la notion de technique est reléguée en dernière position (passant de $12,6 \%$ à $5,2 \%$ d'occurrences) juste après environnement (passé de $12,3 \%$ à $9,1 \%$ d'occurrences) alors que la notion de changement occupe la première place devant durable. De plus, les termes savoir et agriculture ont disparu du noyau central et migré respectivement dans la seconde périphérie et dans les éléments contrastés. Enfin, on note l'apparition des termes éducation, valeur et bien-être dans la 
première périphérie, considérée par Abric (2005) comme une zone potentielle de changement.

Ces résultats témoignent donc d'une modification des représentations initiales du "produire autrement", confirmant l'hypothèse selon laquelle les échanges entre enseignants.es de différents genres et disciplines au cours de l'activité 2 pouvaient induire une évolution dans les représentations. Plus précisément, alors que les enseignants.es assimilaient initialement la notion de "produire autrement» à une forme que l'on pourrait qualifier de «technique pour l'environnement nécessitant un changement dans les territoires et l'alimentation » la représentation finale témoigne d'une vision élargie et plus englobante qui pourrait être résumée comme un « changement durable pour l'environnement par l'éducation et la prise en compte des territoires ». Cette évolution suggère qu'au-delà de définir la notion de "produire autrement", les enseignants.es se sont projetés dans leur rôle vis-à-vis de cette injonction en faisant figurer le terme éducation systématiquement dans la première périphérie, zone correspondant aux termes venant compléter ceux du noyau central. Cela fait écho au fait que l'enseignement, au-delà de contribuer à faire évoluer les pratiques agricoles, poursuit aussi une visée éducative plus large visant l'adoption de comportements responsables qui s'inscrivent dans une pensée critique permettant « la participation et la préparation des élèves au monde actuel et incertain à venir " (Lange, 2015).

51 Ce changement des représentations des enseignants.es est certainement lié à la composition pluridisciplinaire des groupes incluant des participants de différentes disciplines et l'on peut dès lors s'interroger sur l'influence de l'interaction entre enseignants.es dans la structuration des représentations finales. Quoi qu'il en soit il est intéressant de noter que certains écueils ont été évités comme celui d'aboutir à la production d'une vision totalement uniformisée du «produire autrement ». En effet, même si cet exercice a permis d'élargir les représentations initiales des enseignants.es, il n'en reste pas moins que certaines notions ont été renforcées au sein des différents sous-ensembles de la population. Ce résultat suggère que la confrontation entre pairs lors de l'activité 2 a provoqué un élargissement des représentations des enseignants.es à des fins de réflexivité partagée et de négociation sur les éléments investigués.

In fine, les évolutions constatées rejoignent nos hypothèses selon lesquelles les représentations individuelles des enseignants.es du " produire autrement » sont a priori réductrices et qu'il est nécessaire, pour les enrichir, de conduire un travail pluridisciplinaire et associant des enseignants.es novices et expérimentés pour les faire évoluer vers une acception plus globale, favorisant ainsi la construction d'une vision commune. Certes un tel travail doit s'inscrire dans un temps long pour assurer un ancrage et une mobilisation de ces représentations élargies dans les pratiques professionnelles des enseignants.es.

\section{Enseigner à produire autrement : des intentions à la réalité}

53 La volonté institutionnelle affichée de mettre en œuvre la transition agroécologique dans le plan « enseigner à produire autrement " n'échappe pas à un certain nombre de difficultés dans un contexte où les modèles productivistes restent encore très dominants. Dans l'enseignement, et en particulier dans l'enseignement agricole, les concepteurs des référentiels des diplômes sont confrontés à la nécessité d'intégrer les 
questions qui surgissent dans la société et dans le monde professionnel avant même que les savoirs correspondants soient stabilisés. Les enseignants.es ont certainement conscience des changements que le «produire autrement » implique dans les pratiques agricoles et par ricochet, dans les pratiques d'enseignement, mais ils restent avant tout centrés sur les dimensions techniques et environnementales. En effet, peu d'entre eux évoquent les dimensions politiques, sociales, économiques et agronomiques. Or, "produire autrement» implique de donner une large place à l'agroécologie en reconnaissant entre autres les limites des solutions techniques.

Dès lors, les difficultés des enseignants.es sont certainement liées au fait qu'ils sont confrontés à des paradigmes qui nécessitent d'appréhender les différentes dimensions et acceptions de l'agroécologie dont les savoirs associés sont en cours de construction, incertains, instables, complexes, controversés, et dont la pluralité des interprétations est source d'incertitudes tant dans le domaine de la recherche que dans la société. Audelà de l'évolution des représentations, le caractère polysémique de la notion « produire autrement » nécessite des approches plurielles dans la manière de penser et d'agir.

En outre, les référentiels d'aujourd'hui «ne définissent plus de manière aussi précise les notions ou concepts qui font l'objet d'enseignement mais constituent des repères " (Simonneaux et Simonneaux, 2014). Par exemple, dans le référentiel de diplôme du baccalauréat professionnel "conduite et gestion de l'exploitation agricole", l'agroécologie est abordée en proposant de « réaliser une approche pluridisciplinaire intégrative, écologique et agronomique de la notion d'agrosystème " pour répondre à des problèmes empreints d'incertitudes. Dès lors, la traduction de ces notions en objets d'enseignement ne va pas de soi, car elle reste intimement liée à la compréhension et à l'élaboration épistémologique et politique des concepts de l'agroécologie, ce qui n'est pas sans poser de difficultés aux enseignants.es d'autant plus que l'enseignement agricole peine à les traduire pleinement dans ses curricula. Par conséquent, on peut s'attendre à voir apparaître des difficultés dans leur transposition didactique par les enseignants.es.

Cette étude tend à confirmer l'influence de la culture disciplinaire des enseignants.es sur leurs représentations. Dès lors, et du fait de la nature polysémique et complexe des concepts du "produire autrement» et de l'agroécologie, il apparaît nécessaire de favoriser les échanges interdisciplinaires, en proposant des espaces de concertation et de médiation. Ce travail a permis d'engager une réflexion autour des modalités d'action possibles dans la perspective d'enrichir la formation des enseignants.es, en proposant notamment un module dans le cadre du Master Enseignement Éducation et Formation offert par l'ENSFEA. Par exemple, il peut être porteur de mobiliser les représentations identifiées afin de discuter avec les enseignants.es des enjeux et de l'orientation axiologique des concepts qu'ils auront à transmettre à leurs élèves. En effet, ils ont à se questionner sur les savoirs à mobiliser (incertains ou stabilisés) dans un contexte de changement.

Les difficultés des enseignants.es relèvent également en partie de celles rencontrées pour toute réforme scolaire (réactivité, réflexivité, acquisition de connaissances et méthodes nouvelles, etc.). Dès lors, il est certainement illusoire de penser que le changement reposerait avant tout sur la clarté, la pertinence et la légitimité de la prescription, laissant dans l'ombre ce qui dépend de son appropriation par les enseignants.es et du rôle de la formation dans ce processus (Bouillier-Oudot et Asloum, 
2014). Par ailleurs, de nombreux auteurs s'interrogent sur le paradigme même de l'agroécologie et sur sa transposition concrète sur le terrain, dans un contexte où les modèles productivistes restent encore très dominants. Cela nous invite à une certaine vigilance au regard des prescriptions descendantes de l'ÉDD pour laquelle Sauvé (1999) voyait

[...] non pas un changement de paradigme épistémologique, éthique et stratégique, mais une forme progressiste de la modernité, qui vise à préserver les valeurs et les pratiques de cette dernière et qui contribue à promouvoir la rationalité instrumentale où les savoirs scientifiques et techniques sont privilégiés.

In fine, cette étude a permis de caractériser les représentations des enseignants.es au moment du lancement de la Loi d'avenir pour l'agriculture, l'alimentation et la forêt en 2014. Il serait nécessaire à présent, alors que de nouvelles instructions ont été données fin 2019 par le Ministère de l'Agriculture et de l'Alimentation dans le cadre du plan EPA2 « enseigner à produire autrement pour les transitions et l'agroécologie ", d'analyser les effets de la formation offerte sur les représentations des enseignants.es en termes d'ancrage et de mobilisation de ces représentations élargies dans leurs pratiques professionnelles au sein de l'espace scolaire.

\section{BIBLIOGRAPHIE}

Abric, J.C. (1994). L'organisation interne des représentations sociales : système central et système périphérique. Structures et transformations des représentations sociales, 73-84.

Abric, J.C. (2005). Méthodes d'étude des représentations sociales. Toulouse : Éditions érès.

Allaire, G., et Boyer, R. (1995). La grande transformation de l'agriculture : lectures conventionnalistes et régulationnistes. Paris: INRA Éditions.

Altieri, M.A. (1987). Agroecology: the scientific basis of alternative agriculture. Boulder : Westview Press.

Asloum, N. et Kalali, F. (2013). Repères historico critiques de l'évolution des curriculums prescrits de l'enseignement Agricole et de l'Éducation Nationale. Cas de l'éducation au développement durable. Penser l'éducation, Hors-série 2013, 449-466.

Barthes, A., Lange, J.-M., Tutiaux-Guillon, N. (2017). Dictionnaire critique des enjeux et concepts des "éducations à ». Paris : L'Harmattan.

Bouillier-Oudot, M. H. et Asloum, N. (2014). Une approche curriculaire de l'évolution de l'offre de formation de l'enseignement technique agricole au regard des attentes sociales en matière d'environnement. Dans P. Orly et J.F. Marcel (dir.). Recherches en éducation : pratiques et apprentissages professionnels (p. 95-111). Dijon : Éducagri éditions.

Boulet, M. (2003). La formation des acteurs de l'agriculture en France : continuités et ruptures, 1945-1985. Dans M. Boulet (dir.). Actes du colloque, ENESAD, 27-29 novembre 2001. Dijon : Éducagri éditions.

Flament, C. (1962). L'analyse de similitude. Cahiers du centre de recherche opérationnelle, 4, 63-97. 
Flament, C. (1994a). Aspects périphériques des représentations sociales. Structures et transformations des représentations sociales, 85-118.

Flament, C. (1994b). Le plaisir et la rémunération dans la représentation sociale du travail. Cahiers Internationaux de Psychologie Sociale, 23, 61-69.

Francis, C., Lieblein, G., Gliessman, S., Breland, T. A., Creamer, N., Harwood R., Salomonsson, L., Helenius, J., Rickerl, D., Salvador, R., Wiedenhoeft, M., Simmons, S., Allen, P., Altieri, M., Flora, C. et Poincelot, R. (2003). Agroecology: the ecology of food systems. Journal of Sustainable Agriculture, 22(3), 99-118.

Fruchterman, T. et Reingold, B. (1991). Graph drawing by force-directed placement. SoftwarePractice and Experience, 21, 1129-1164.

Garnier, C. et Sauvé, L. (1999). Apport de la théorie des représentations sociales à l'éducation relative à l'environnement - Conditions pour un design de recherche. Éducation relative à l'environnement : Regards - Recherches - Réflexions, 1, 65-77.

Girault, Y., Lange, J.M., Fortin-Debart, C., Simonneaux, L. et Lebeaume, J. (2007). La formation des enseignant.e.s dans le cadre de l'éducation à l'environnement pour un développement durable : problèmes didactiques. Éducation relative à l'environnement : Regards - Recherches - Réflexions, 6, 119-136.

Girvan, M., et Newman, M.E.J. (2002). Community structure in social and biological networks. Proceedings of the National Academy of Sciences of the United States of America, 99, 7821-7826.

Gliessman, S.R. (1990). Agroecology: researching the ecological basis for sustainable agriculture. In Gliessman S.R. (eds). Agroecology. Ecological Studies (Analysis and Synthesis) (p 3-10). New York: Springer.

Hazard, L., Monteil, C., Duru, M., Bedoussac, L., Justes, E., Theau, J.P. (2016). Agroécologie. Dictionnaire d'Agroécologie. Récupéré de https://dicoagroecologie.fr/encyclopedie/agroecologie/

Jodelet, D. (1989). Folies et représentations sociales. Paris : Presses Universitaires de France.

Jodelet, D. (2003). Représentations sociales : un domaine en expansion. Dans Jodelet D. (dir.). Les représentations sociales (p. 45-78). Paris : Presses Universitaires de France.

Lange, J.M. (2011). Éducation au développement durable : éléments pour une problématisation de la formation des enseignant.e.s. Carrefours de l'éducation, 1(3), 71-85.

Lange, J.M. (2015). Éducation et engagement : La participation de l'École à relever les défis environnementaux et de développement. Éducation relative à l'environnement, 12.

Legardez, A. (2004). L'utilisation de l'analyse des représentations sociales dans une perspective didactique : l'exemple de questions économiques. Revue des sciences de l'éducation, 30(3), 647-665.

Loubère, L. et Ratinaud, P. (2014). Documentation IRaMuTeQ 0.6 alpha 3 version 0.1. Récupérée le 23 janvier 2020 de www.iramuteq.org/documentation/fichiers/documentation_19_02_2014.pdf

Mabit, R. (1991). Formations à l'environnement : l'expérience de l'enseignement du Ministère de l'Agriculture. Aménagement et Nature, 101, 8-10.

Martinand, J.L. (1989). Pratiques de référence, transposition didactique et savoirs professionnels en sciences techniques. Les sciences de l'éducation, pour l'ere nouvelle, 2, 23-29.

Martinand, J.L. (2003). L'éducation technologique à l'école moyenne en France : problèmes de didactique curriculaire. La revue canadienne de l'enseignement des sciences, des mathématiques et des technologies, 3, 100-116. 
Ministère de l'agriculture et de l'alimentation. (2019a). Infographie - Les chiffres de la rentrée 2019 dans l'enseignement agricole mis à jour le 30 août 2019. Récupéré le 07 septembre 2020 de https:// agriculture.gouv.fr/infographie-les-chiffres-de-la-rentree-2019-dans-lenseignement-agricole

Ministère de l'Agriculture et de l'Alimentation. (2019b). Référentiel de diplôme du baccalauréat professionnel de la spécialité « conduite et gestion de l'entreprise agricole » mis à jour le 7 novembre 2019. Récupéré le 23 janvier 2020 de https://chlorofil.fr/fileadmin/user_upload/02-diplomes/ referentiels/secondaire/bacpro/cgea/bac-pro-cgea-ref-012020.pdf

Ministère de l'Agriculture et de l'Alimentation. (2020). Tutorat des Agents Contractuels enseignants du Ministère de l'Agriculture et de l'Alimentation. Récupéré le 02 septembre 2020 de https:// chlorofil.fr/fileadmin/user_upload/01-systeme/emplois/tutac/2020/tutac2020-s3.pdf

Moliner, P. (1993). Cinq questions à propos des représentations sociales. Cahiers internationaux de psychologie sociale, 20, 5-14.

Moscovisi, S. (1961). La psychanalyse, son image et son public. Paris : Presses Universitaires de France.

Muller, P. (2000). La politique agricole française : l'État et les organisations professionnelles. Économie rurale, 255(1), 33-39.

Poly, J. (1978). Pour une agriculture plus économe et plus autonome. https://hal.inrae.fr/view/index/ identifiant/hal-02859092

Sauvé, L. (1999). L'éducation relative à l'environnement entre modernité et postmodernité. Les propositions du développement durable et de l'avenir viable. Canadian Journal of Environmental Education, 4, 9-35.

Simonneaux, L. et Simonneaux, J. (2014). The emergence of recent science education research and its affiliations in France. Perspectives in Science, 2(1-4), 55-64.

Stassart, P.M., Baret, P., Grégoire, J.C., Hance, T., Mormont, M., Reheul, D. et Visser, M. (2012). L'agroécologie : trajectoire et potentiel pour une transition vers des systèmes alimentaires durables. Dans Van Dam, D., Nizet, J. et Streith, M. (dir.). Agroécologie. Entre pratiques et sciences sociales (p. 25-51). Dijon : Educagri éditions.

Tomich, T.P., Brodt, S., Ferris, H., Galt, R., Horwath, W.R., Kebreab, E., Leveau, J.H., Liptzin, D., Lubell, M., Merel, P., Michelmore, R., Rosenstock, T., Scow, K., Six, J., Williams, N. et Yang, L. (2011). Agroecology: a review from a global-change perspective. Annual Review of Environment and Resources, 36, 193-222.

Vergès, P., (1994). Approche du noyau central : propriétés quantitatives et structurales. Structures et transformations des représentations sociales, 277, 233-53.

Wezel, A., Bellon, S., Doré, T., Vallod, D. et David, C. (2009). Agroecology as a science, movement or practice. Agronomy for Sustainable Development, 29, 503-515.

\section{RÉSUMÉS}

Le projet agro-écologique (loi $\mathrm{n}^{\circ}$ 2014-1170), mobilisant les acquis de l'éducation au développement durable, engage l'enseignement agricole français sur la voie de transitions écologiques, pédagogiques et éducatives ("enseigner à produire autrement»). Face à ces directives, les enseignants.es se heurtent à diverses difficultés, notamment dans la définition des concepts à mobiliser qui impliquent des savoirs de références pluriels, incertains et parfois controversés. Par une série d'évocations libres et hiérarchisées, cette recherche conduite auprès 
de 340 enseignants.es vise à rendre compte de leurs représentations du "produire autrement ». En mobilisant la théorie des représentations sociales, les résultats montrent que celles-ci sont initialement étroites et techniques, avec des éléments partagés et d'autres susceptibles de variations. Cependant, elles évoluent et s'enrichissent par des échanges entre pairs, pour donner au final une place forte à l'éducation. Il devient urgent de sensibiliser les enseignants.es à la réflexion sur les fondements de ces changements programmés en intégrant une analyse sociale et épistémologique des savoirs en jeu.

The agro-ecological project (law ${ }^{\circ} 2014-1170$ ), mobilizing the achievements of education for sustainable development, sets French agricultural education on the path of ecological, pedagogical and educational transitions ("teaching to produce differently"). Faced with these directives, teachers encountered various difficulties, particularly in defining the concepts to be mobilized, which involve plural, uncertain and sometimes controversial knowledge. Through a series of free and hierarchical evocations, this research conducted among 340 teachers aims to give an account of their representations of "to produce differently". By mobilizing the theory of social representations, the results show that they are initially restrictive and technical, with some elements shared and others susceptible to variation. However, they evolve and are enriched by exchanges between peers, ultimately giving a strong place to education. It is becoming urgent to sensitize teachers to reflect on the foundations of these programmed changes by integrating a social and epistemological analysis of knowledge.

\section{INDEX}

Mots-clés : agroécologie, enseignement agricole, représentation sociale, analyse de similitude, analyse prototypique, analyse lexicale

Keywords : agroecology, agricultural education, social representation, similarity analysis, prototypical analysis, lexical analysis

\section{AUTEURS}

\section{NINA ASLOUM}

Maître de conférences en sciences de l'éducation et de la formation à l'École Nationale Supérieure de Formation de l'Enseignement Agricole et membre de l'UMR Éducation, Formation, Travail, Savoirs, Université de Toulouse, elle coordonne la formation des enseignant.e.s en aménagements des espaces. Co-responsable du Master Enseignement Éducation et Formation depuis 2019. Ses recherches portent sur les pratiques enseignantes en questionnant la manière dont les enseignant.e.s se saisissent de nouvelles prescriptions relatives à l'éducation au développement durable. Courriel : nina.asloum [@] ensfea.fr

\section{LAURENT BEDOUSSAC}

Maître de conférences HDR en agronomie à l'École Nationale Supérieure de Formation de l'Enseignement Agricole, il coordonne la formation des enseignant.e.s en productions horticoles et viticulture-œnologie. Depuis 2014, il est co-responsable du Master of Science AgroFoodChain. Il conduit ses recherches à l'Institut national de recherche pour l'agriculture, l'alimentation et l'environnement dans l'UMR AGIR sur l'analyse des performances et du fonctionnement des associations d'espèces pour la conception de systèmes de culture et de filières plus agroécologiques. Courriel : laurent.bedoussac [@] ensfea.fr 